\title{
Demand Response and Building Energy Simulation
}

\author{
April 10, 2012
}

Douglas Black, Sila Kiliccote, Mary Ann Piette, and Rongxin Lin

Lawrence Berkeley National Laboratory

Demand Response Research Center

http://drrc.lbl.gov/

Sponsored by California Energy Commission
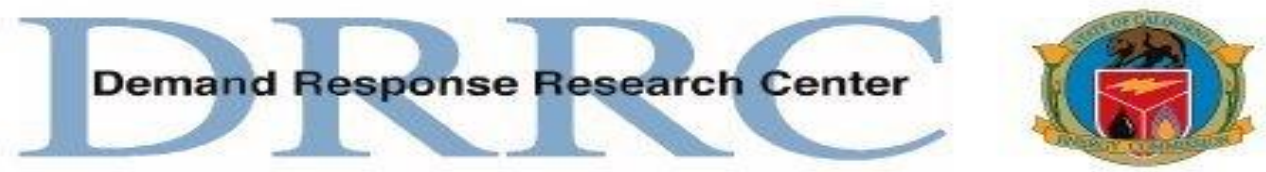


\section{What is Demand Response?}

- Definition of Demand Response (DR): Changes in electric usage by demand-side resources from their normal consumption patterns in response to changes in the price of electricity over time, or to incentive payments designed to induce lower electricity use at times of high wholesale market prices or when system reliability is jeopardized.

- The estimated minimum amount of power electricity consumers demand on a day-to-day basis is called the baseload.

- Demand for electric power is highest in the afternoon and early evening. The periods when demand is greatest are called peak usage times.

- Dynamic pricing, time-of-use rates (TOU) and upgrading to smart grid technology are all examples of demand response programs that could reduce energy consumption, reduce power outages and reduce wholesale energy costs.

- DR addresses peak demand (kW) and not overall consumption (kWh).

- Ultimately, with successful DR, fewer power plants are needed. 


\section{Demand Response Research Center}

- The California Energy Commission's Public Interest Energy Research (PIER) program's Demand Response Research Center (DRRC) is led by Lawrence Berkeley National Laboratory; http://drrc.lbl.gov/

- The main objective of the DRRC is to develop, prioritize, conduct, and disseminate research that develops broad knowledge that facilitates DR

- "Demand Shifting with Thermal Mass" is one of the research projects conducted by DRRC; it investigates the methods and effects of shifting electrical energy usage in small and large commercial buildings using various pre-cooling strategies 


\section{Demand Response Research Center}

1. Electric Systems and Strategic Issues

- Valuing Demand Response

- Dynamic Tariffs, Rate Design, Ancillary Services

- Communications and Telemetry

2. Buildings

- Automation, Communications and Control

- End-Use Control Strategies and Models

- Behavior-response to dynamic tariffs

3. Industry

- Automation, End-Uses and Controls

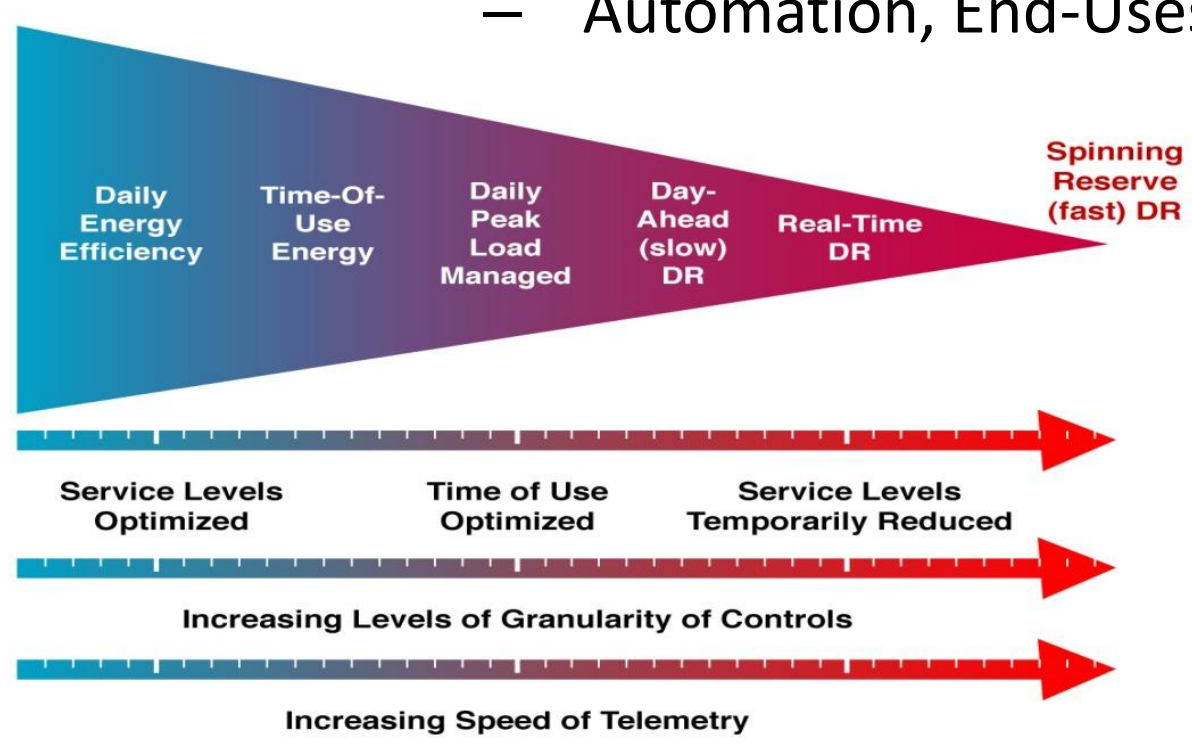




\section{DRRC Research Portfolio}

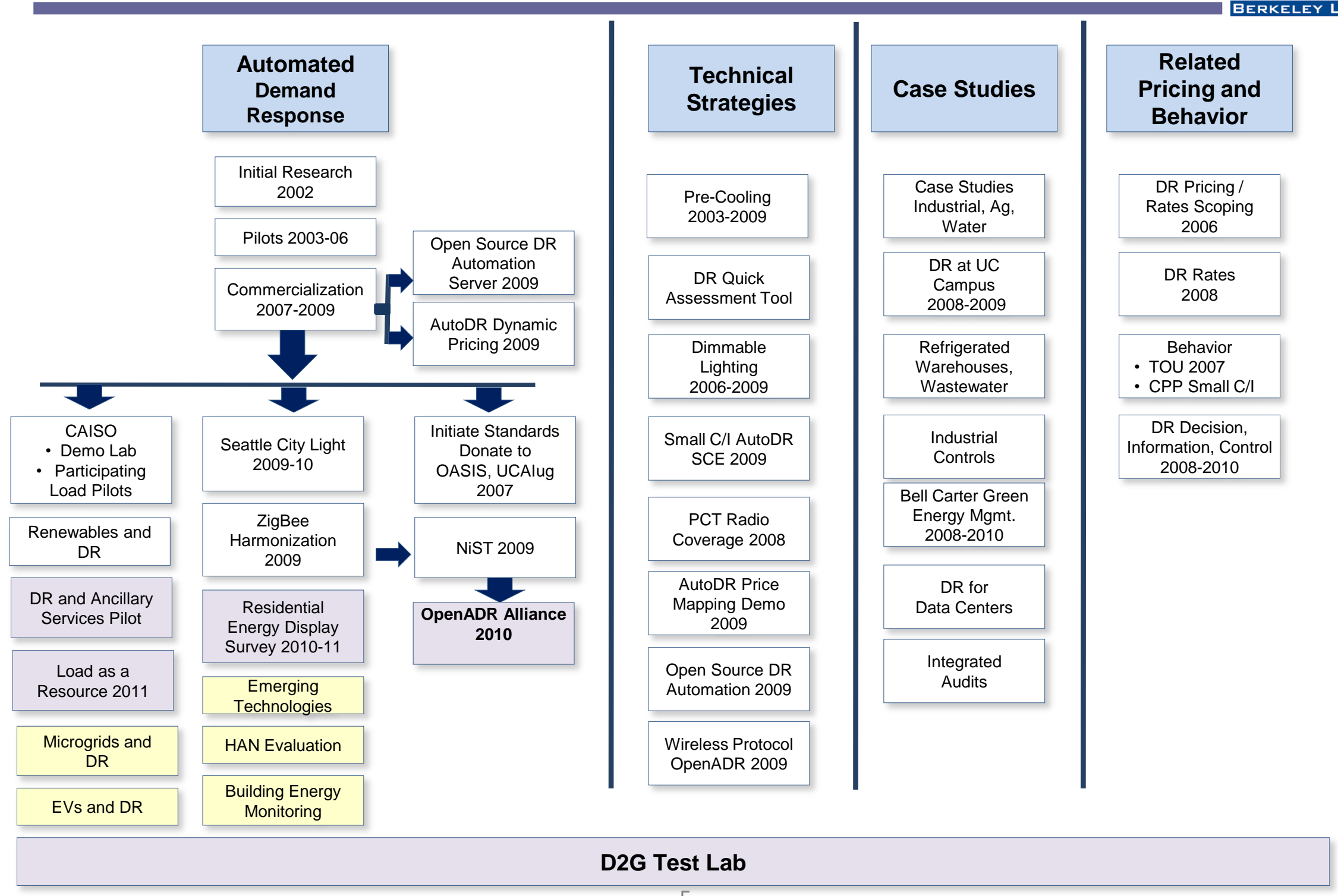




\section{OpenADR is becoming a National Standard}

\section{First official OpenADR v1.0}

specification by LBNL/CEC*

Research initiated by LBNL/ DRRC

(California Energy Commission PIER)

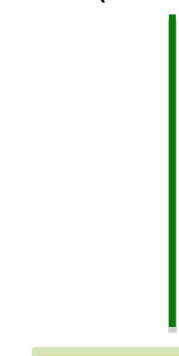

Pilots and field trials

- 2003: Developments, tests

- 2004: Scaled-up tests, relay

- 2005-06: CPP/ Auto-CPP (PG\&E)
OpenADR Commercialization

(PG\&E, SCE, and SDG\&E)
1. OpenADR standards

2. Pilots and field trials

- Wholesale markets (CAISO)

- Pacific-NW (Winter DR)

3. International demos.

*OpenADR v1.0: http://openadr.lbl.gov/

1. OpenADR donated to OASIS and UCAlug

- UCA OpenADR Taskforce formed - OASIS EI TC formed

2. NIST Smart Grid, PAP 09

3. Honeywell Smart Grid - ARRA 80MW Auto-DR w/ SCE

\section{1}
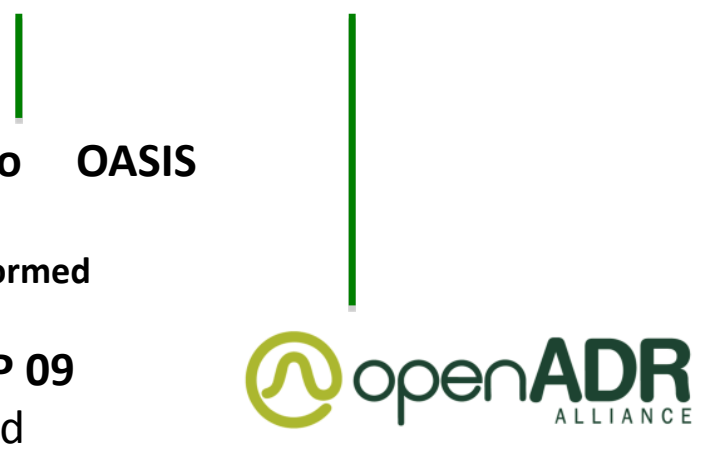

Over $250 \mathrm{MW}$ of DR in California from AutoDR 


\section{Historic focus on Seasonal Grid Stress}

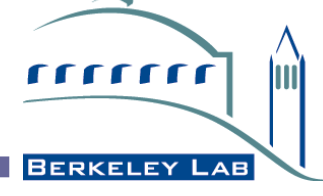

\section{OpenADR PG\&E Demand Bid Test Day}

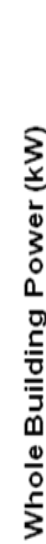

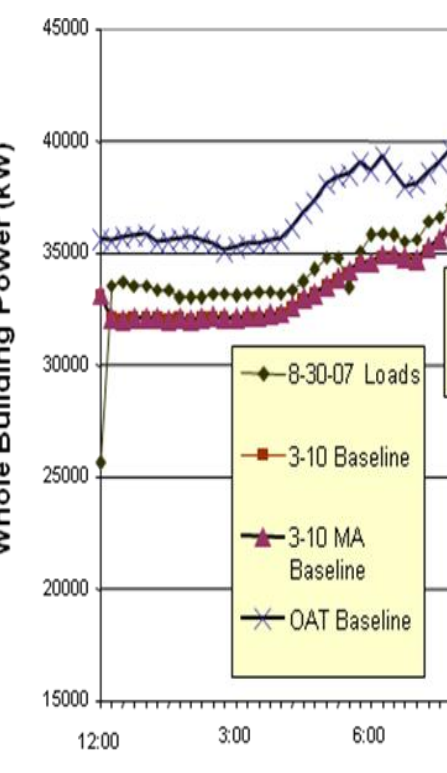

Extensive R\&D on baselines Future directions ancillary services

$6: 00$
OpenADR Northwest Test on Cold Morning

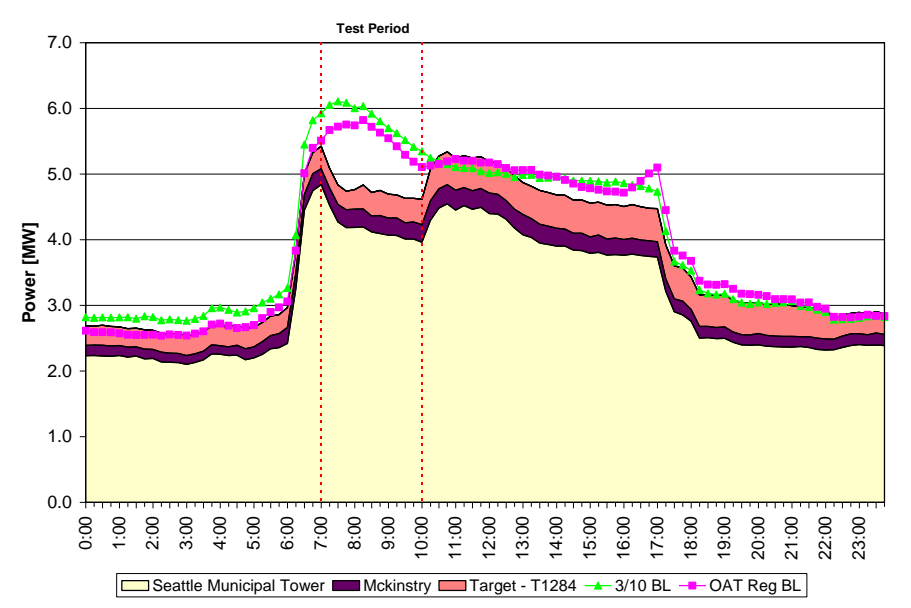

OpenADR Cumulative Shed in July 2008

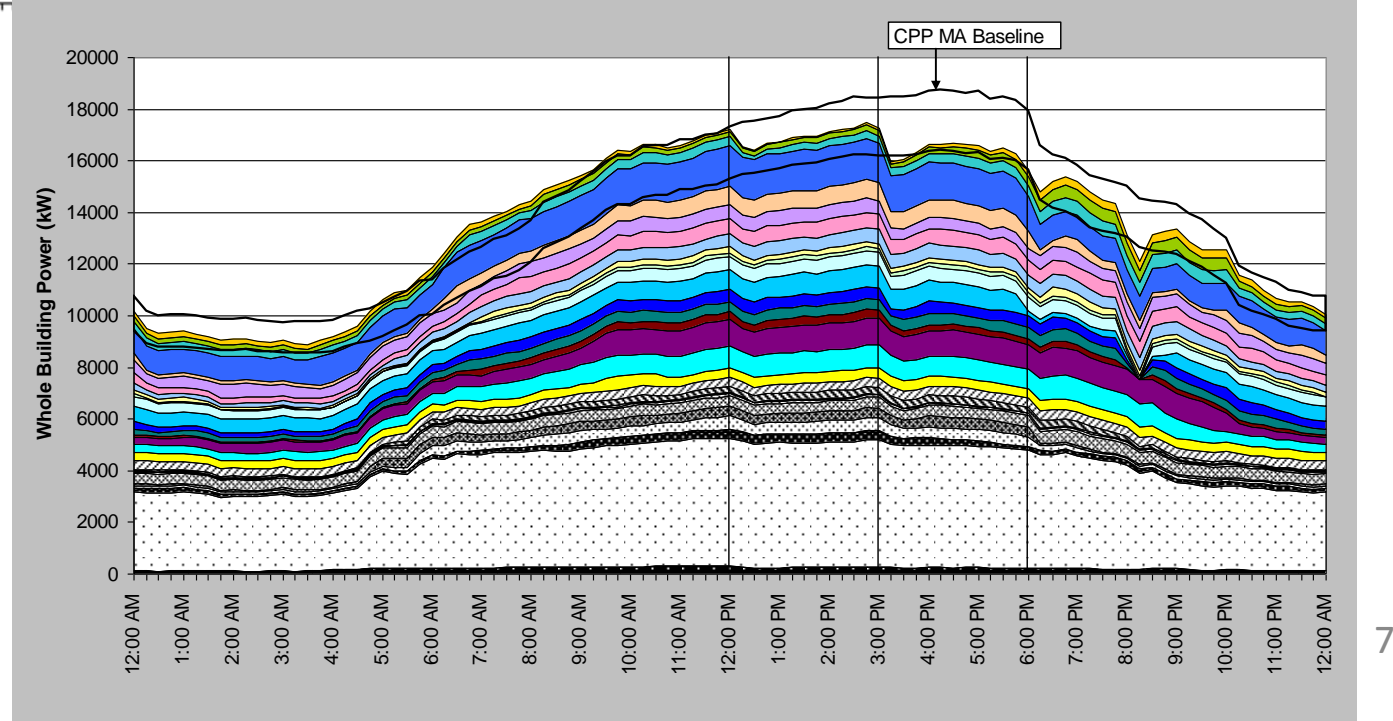




\section{DR Control Strategies Evaluated in Previous Research}

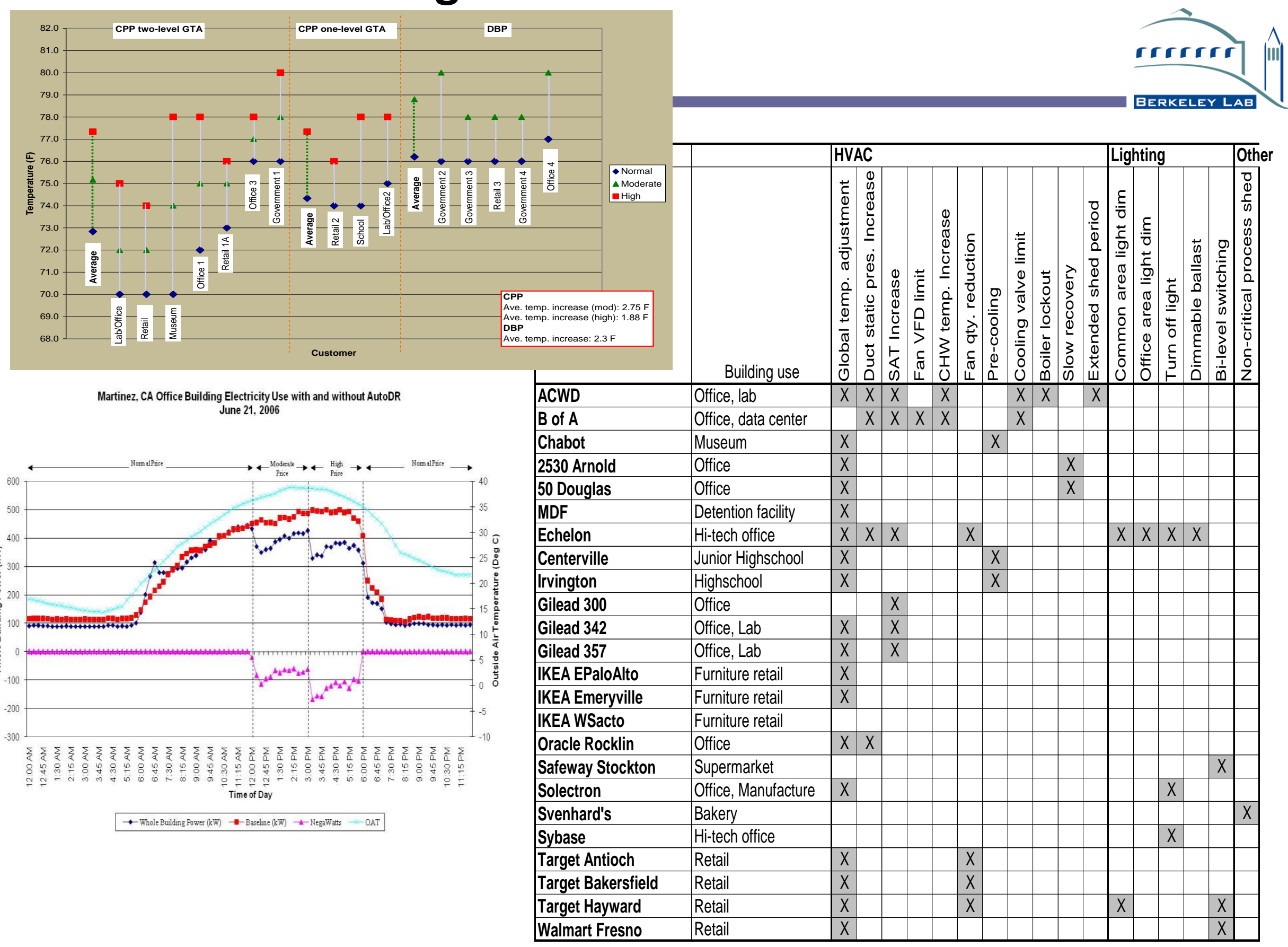




\section{In Summary DRRC is ...}

- Continuing to pursue its vision:

- Efficiency and demand response fully integrated under a unified default tariff / incentive structure.

- All customers, all load participates.

- Major appliances come "DR Ready" from the factory.

- All buildings are "DR Enabled" .

- Full automated system integration between generators, distributors and customers.

- Expanding research:

Basic Research

- Load Analysis methods, DR forecasting algorithms, DR impact analysis methods, physical and statistical modeling of resources etc.

\section{Applied Research}

- Tools and Guides

- Residential Energy Display Systems

- Technologies for Small Commercial Customers

- Distribution Automation integration with DR

- Renewables and DR integration

- Collaboration with IOUs, national and international research and development groups

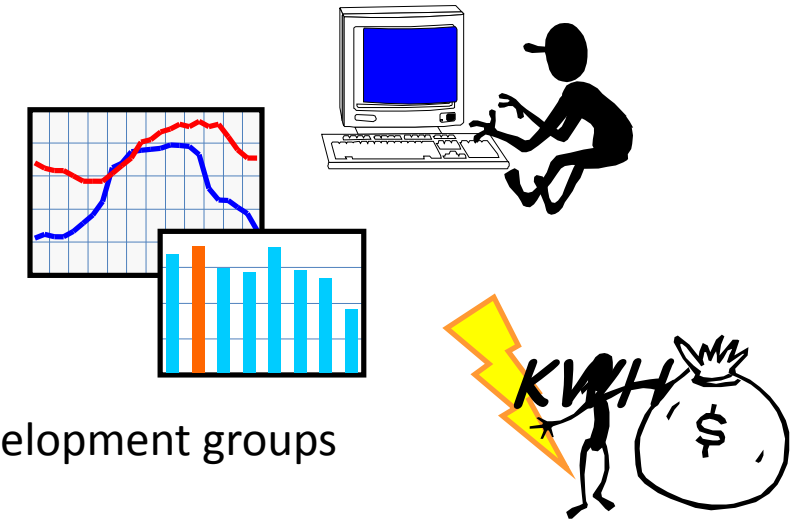

- Seeking collaboration opportunities 


\section{Introduction to DRQAT}

-DRQAT (Demand Response Quick Assessment Tool) is a tool for simulating large commercial buildings developed by LBNL

-It is a user-friendly "wrapper" around the EnergyPlus building energy simulation program.

- Large commercial buildings differ from small commercial building in terms of building materials and size, HVAC equipment, and utility rates - It uses prototypical models of buildings and HVAC equipment -Allows the user to specify a relatively small number of important parameters in order to determine a quick assessment of a range of demand response strategies

-The critical outputs are predictions of energy demand, utility costs, and occupant comfort 


\section{DRQAT Prototype Models}

- DOE reference models of office and retail buildings

- Parameters modified to meet California Title 24 energy and building code

- Building envelope and window thermal properties

- Minimum outdoor air flow rate requirements

- Cooling plant performance parameters

- EnergyPlus auto-sizing adjusts cooling plant capacity to meet design-day weather conditions 


\section{Prototypical Office Building Model}

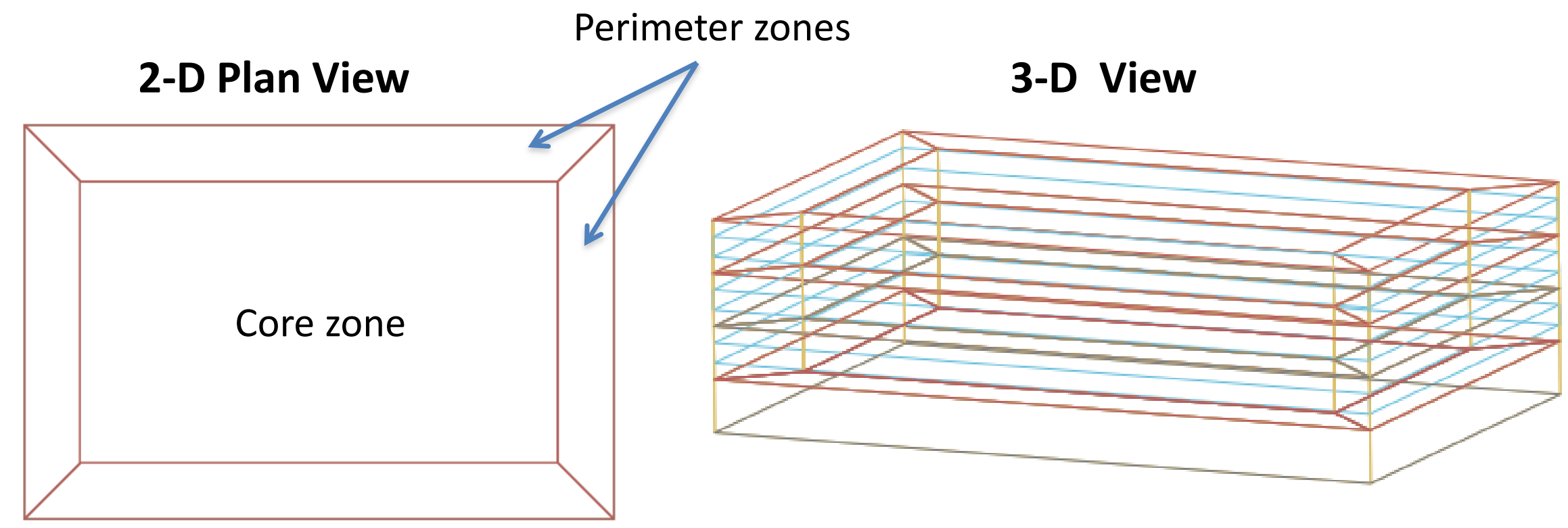

- HVAC Model Description

- Electric Chiller + VAV system

- Rooftop Unit + VAV system

- Airflow and capacity are auto-sized by EnergyPlus 


\section{Prototypical Retail Building Model}

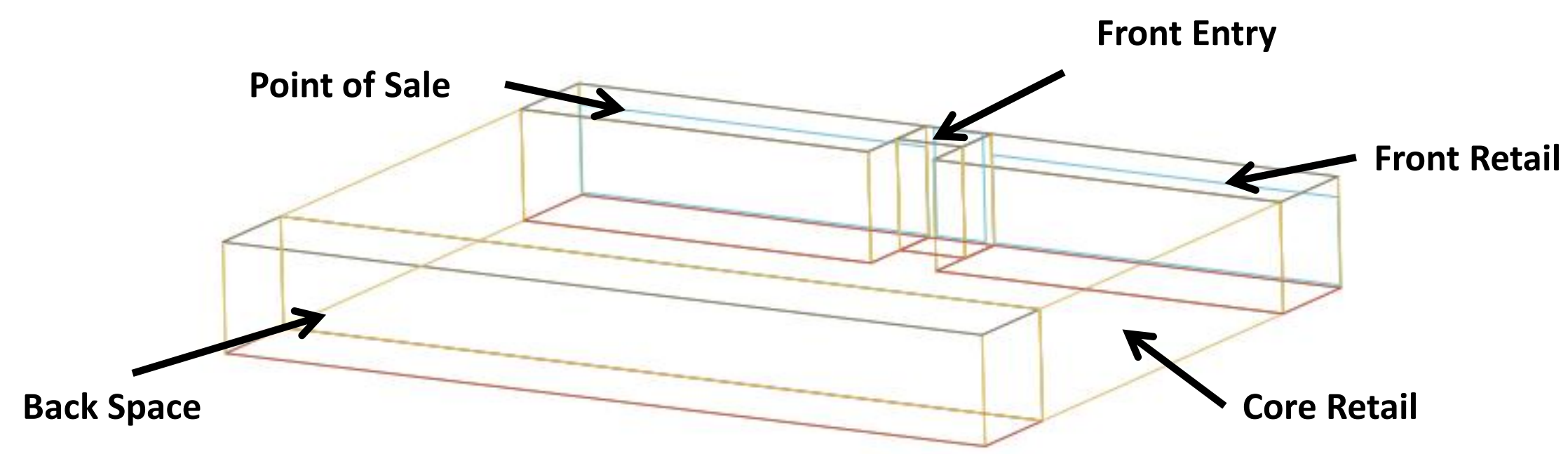

- HVAC Model Description

- Electric Chiller + VAV system

- Rooftop Unit + VAV system

- Airflow and capacity are auto-sized by EnergyPlus 


\section{DRQAT Building Inputs/Outputs}

- DRQAT Inputs

- Building construction parameters

- Utility rates-All California rates are pre-programmed; user can enter custom utility rate structures

- Cooling system parameters

- Internal loads - lights, plug, occupancy-peak values and hourly schedules

- DR strategies - changes to settings and schedules to implement on DR days

- Weather data (OAT, solar radiation, $\mathrm{RH}$ ) from typical meteorological year (TMY3), which is not average, but most frequent temperature for a given hour on a given day for past 30 years

- DRQAT Outputs

- Demand reduction

- Cost savings

- Occupant comfort

- DRQAT advanced users experienced with EnergyPlus can do more with the EnergyPlus input and output files generated by DRQAT. 


\section{DRQAT Building Inputs}
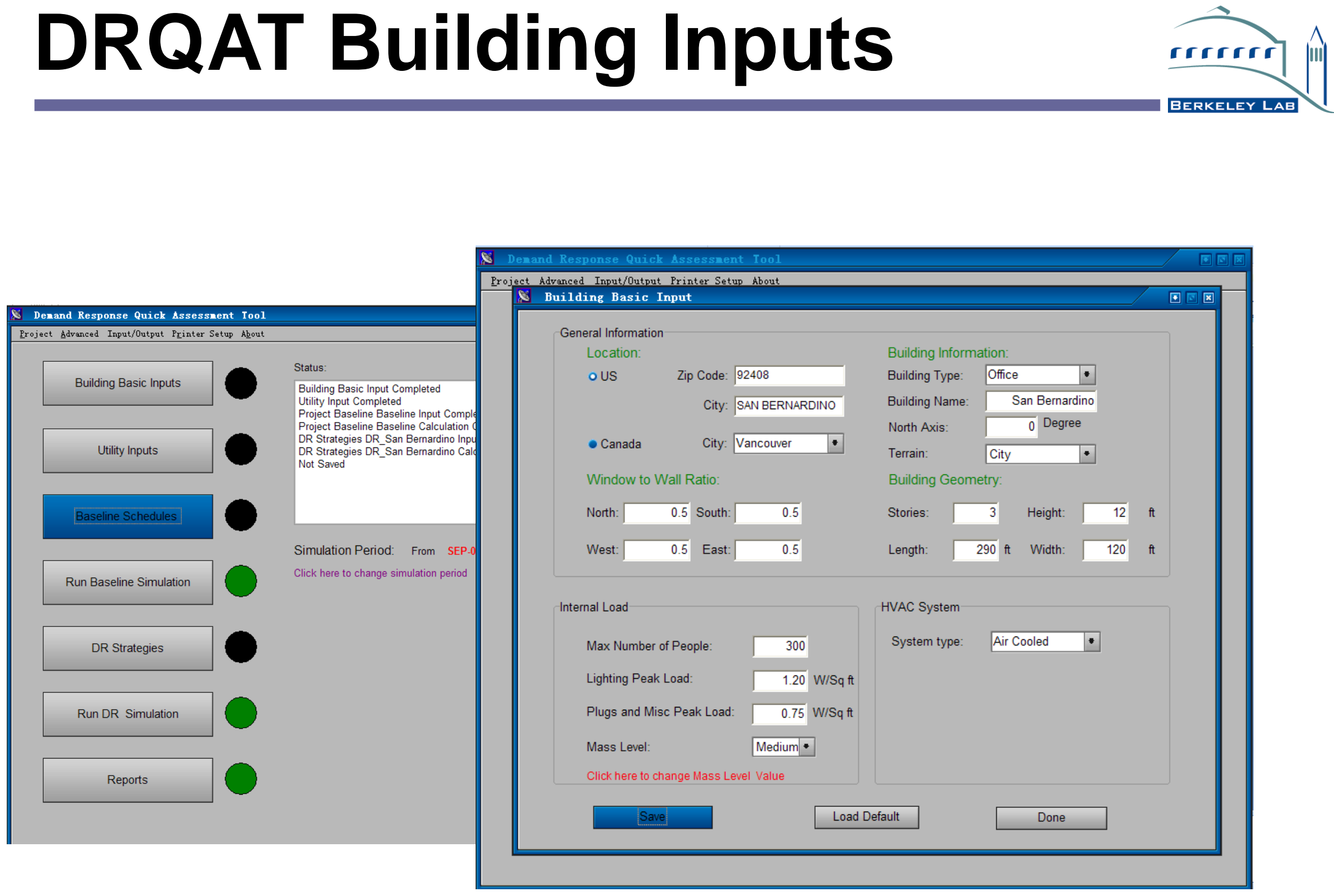


\section{DRQAT - Utility Inputs}
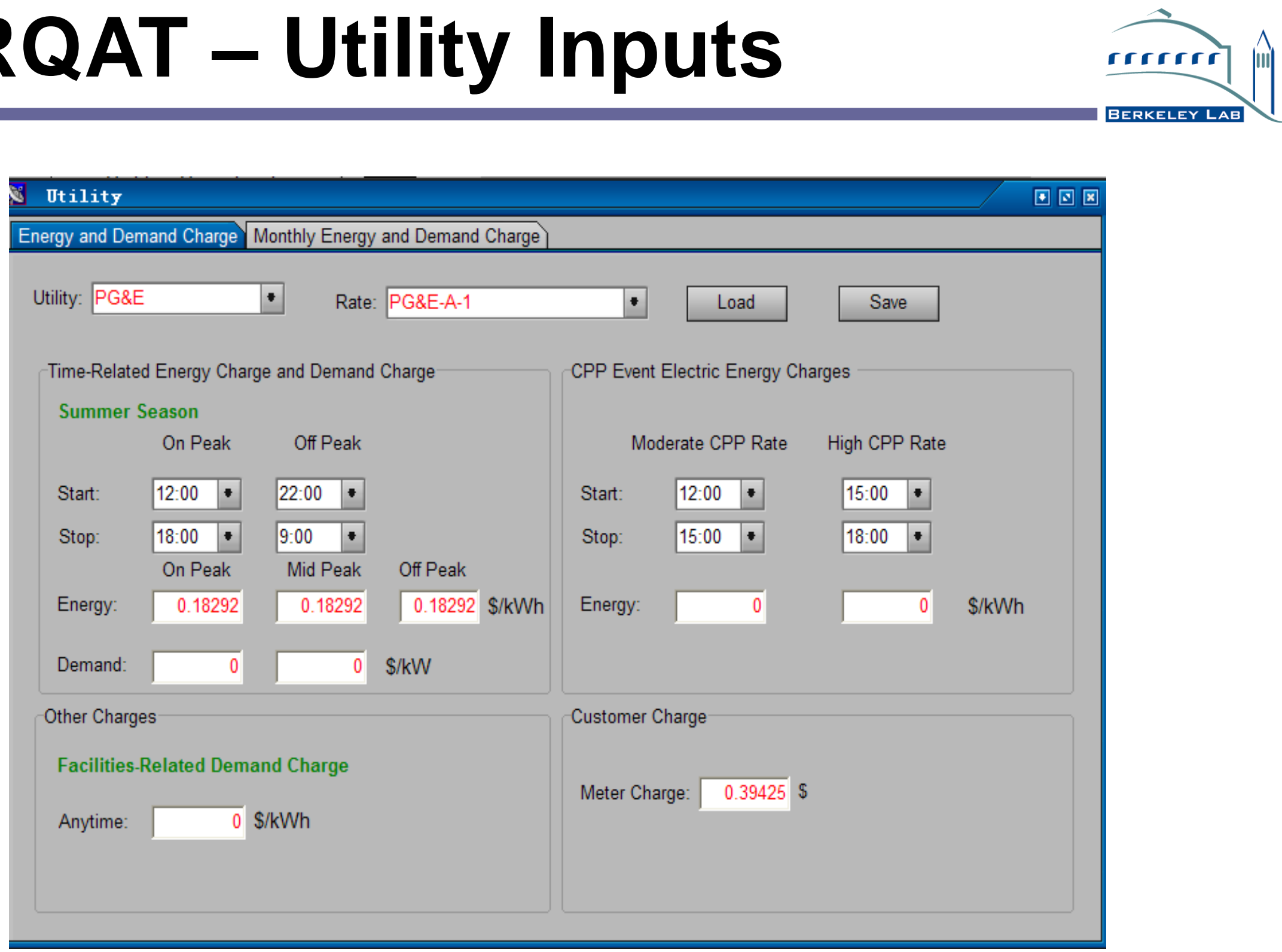


\section{DRQAT - Zone Temp Schedule}

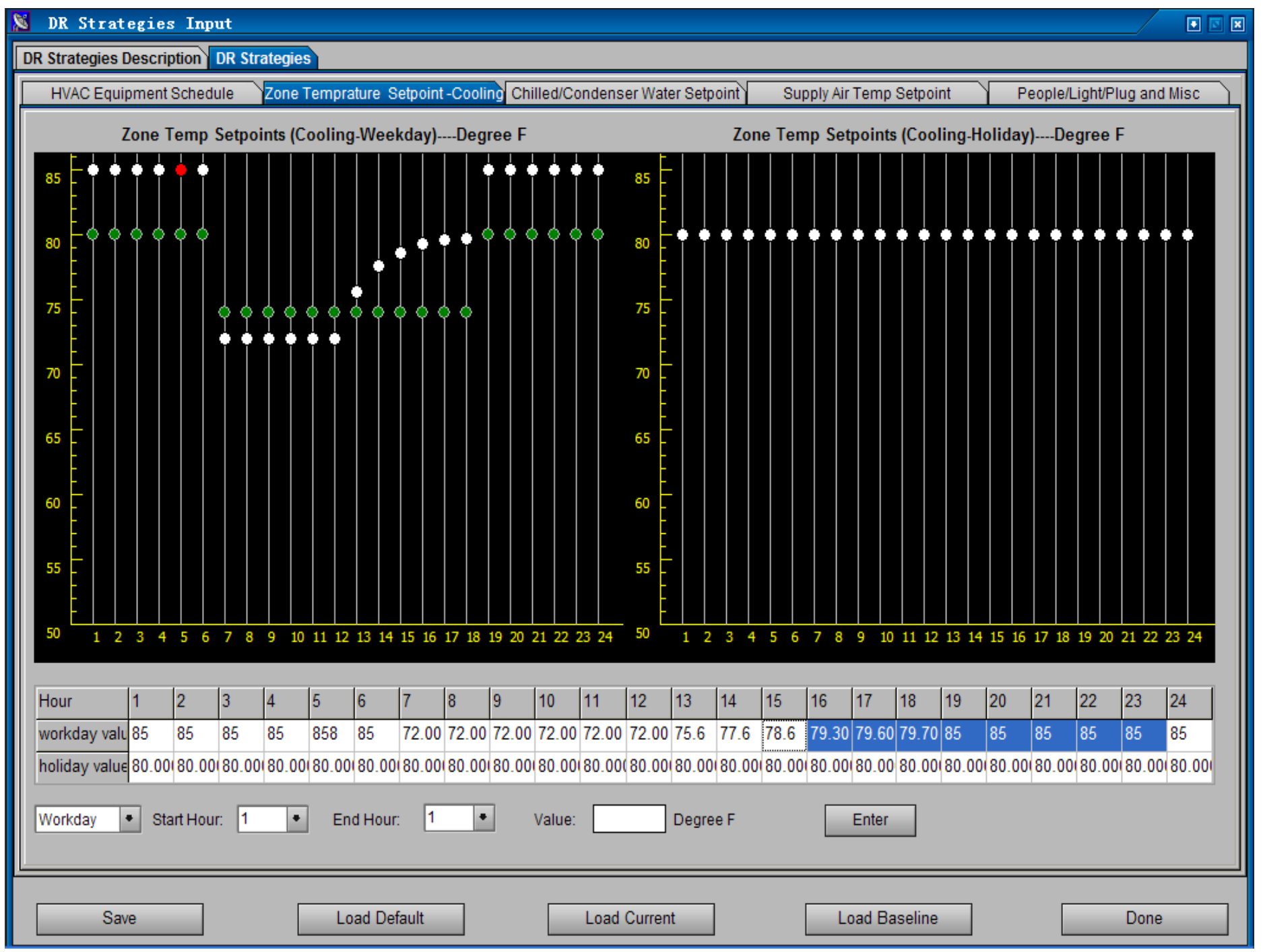




\section{DRQAT - Output of Average Zone Temp and Whole Building Electric Demand}

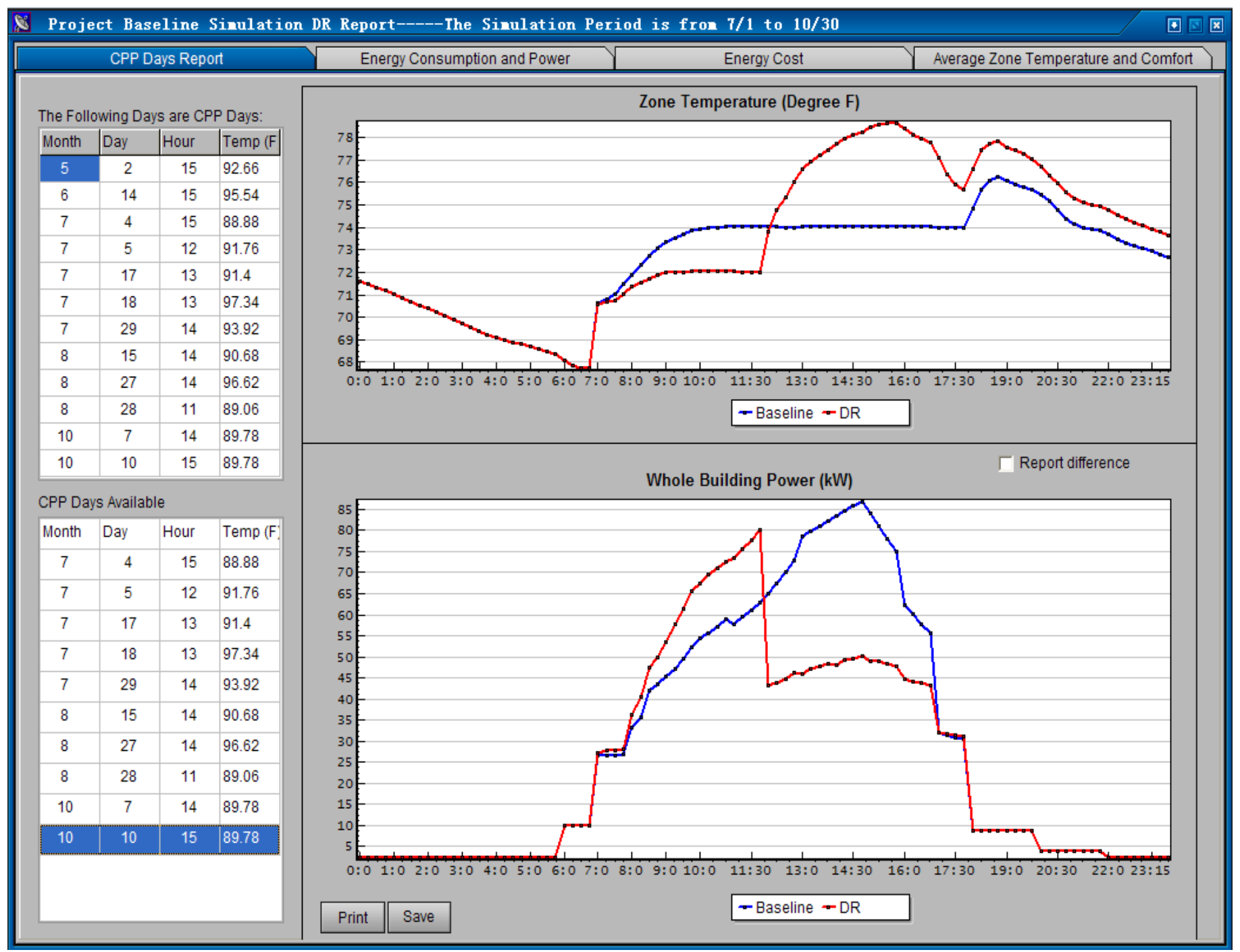




\section{DRQAT Performance}

- Utility (PG\&E) compared DRQAT predicted demand reductions to the actual measured demand reductions at $\sim 40$ commercial buildings

- The average demand reduction over a season predicted by DRQAT was within $5 \%$ for $85 \%$ of the sites. 


\section{DRQAT Input Data Sources}

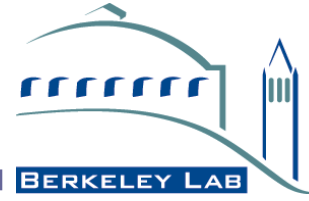

Data Collection $\square$ Input data into the model $\square$

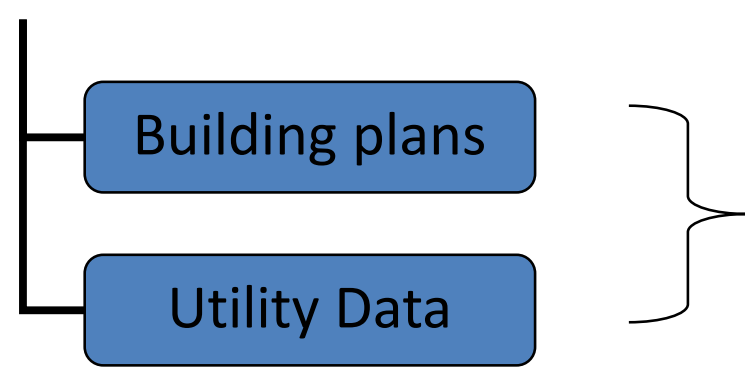

On- Site Surveys

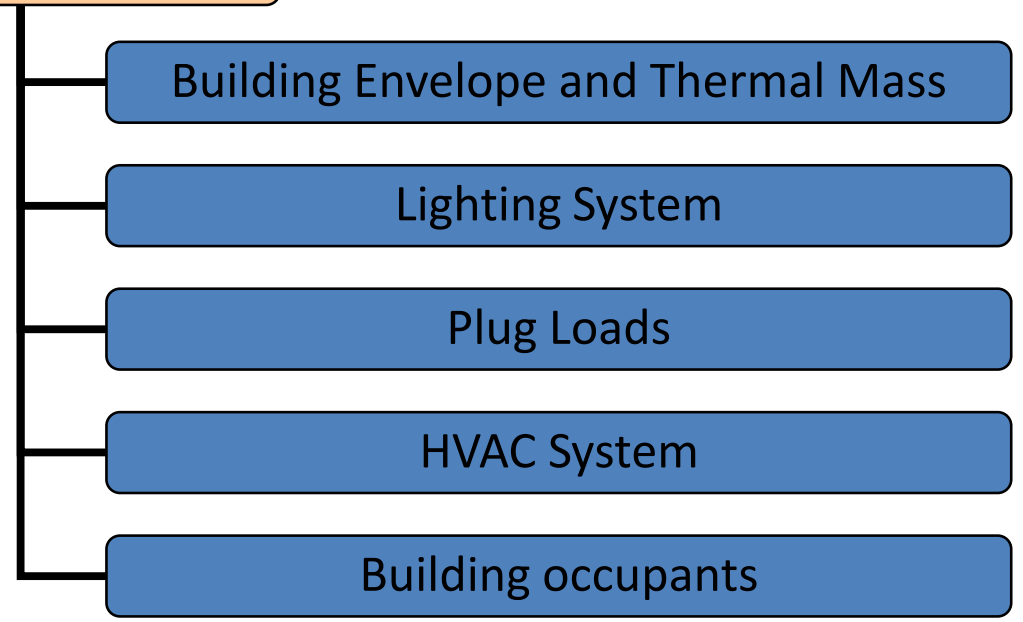




\section{DRQAT Model Inputs}

\section{Data Collection $\square$ Input data into the model $\square$}

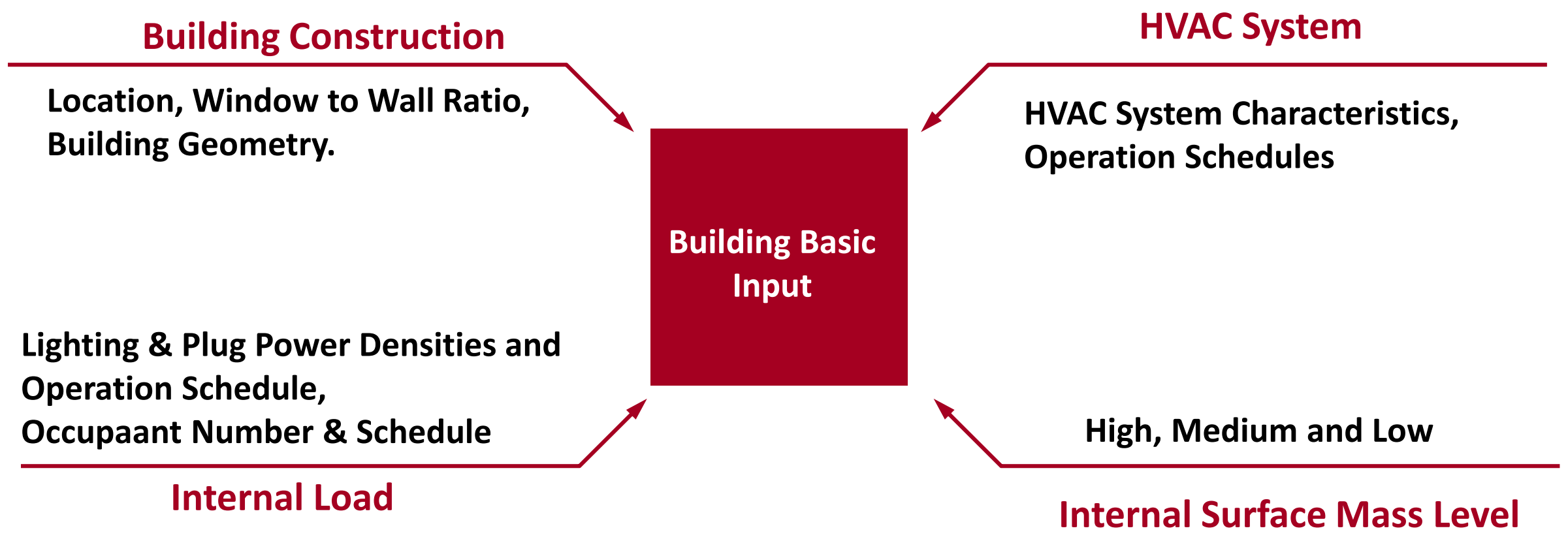




\section{DRQAT Model Calibration}

Input Data into the Model $\square$ Calibration $\square$

1. Enter the actual weather data measured at or near the building into the model;

2. Compare the simulated energy consumption to the measured energy usage data; monthly utility bills and hourly data 


\section{Model Calibration Criteria}

Input Data into the Model $\square$ Calibration

1. Acceptable tolerance for monthly data calibration

\begin{tabular}{|l|l|}
\hline Index & Monthly \\
\hline MBE & $\pm 5 \%$ \\
\hline CV(RMSE) & $\pm 15 \%$ \\
\hline
\end{tabular}

ASHRAE'S GUIDELINE 14-2002

FOR MEASUREMENT OF

ENERGY AND DEMAND

SAVINGS

2. Daily and hourly agreement

\begin{tabular}{|l|c|c|}
\hline Index & Daily & Hourly \\
\hline MBE & $\pm 10 \%$ & $\pm 20 \%$ \\
\hline
\end{tabular}

* $\pm 20 \%$ for a minimum of 20 out of 24 hours for each day

* MBE: Mean bias error

* CV(RMSE): Coefficient of Variation of the Root Mean Square Error 


\section{Steps Following Calibration}

Calibration

\section{Refine the model}

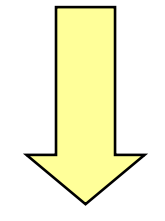

Confirm the baseline model

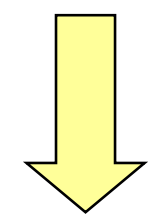

DR simulation

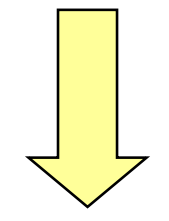

Calculate Saving

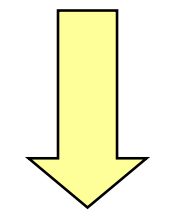

Report 


\section{Optimal Pre-cooling Strategy Study}

- Pre-cooling refers to a demand response strategy that uses a building's thermal mass to shift demand from peak afternoon periods to earlier in the day.

- Pre-cooling is done by lowering the building's temperature set-points in the morning (typically 5 AM to 12 PM).

- The demand savings are achieved by raising the building's temperature set-points in the afternoon.

- Key to success is meeting the demand reduction goal while not making the occupants too cold in the morning and/or too hot in the afternoon.

- Studies of optimal temperature adjustment strategies (e.g. single step, multiple step, exponential increase) and key building parameters have been conducted 


\section{Building Thermal Mass}

- Thermal Capacity

- Primarily determined by the mass of construction materials (exterior \& interior walls, roof, floor) and internal mass

- Thermal Mass in Prototypical Model

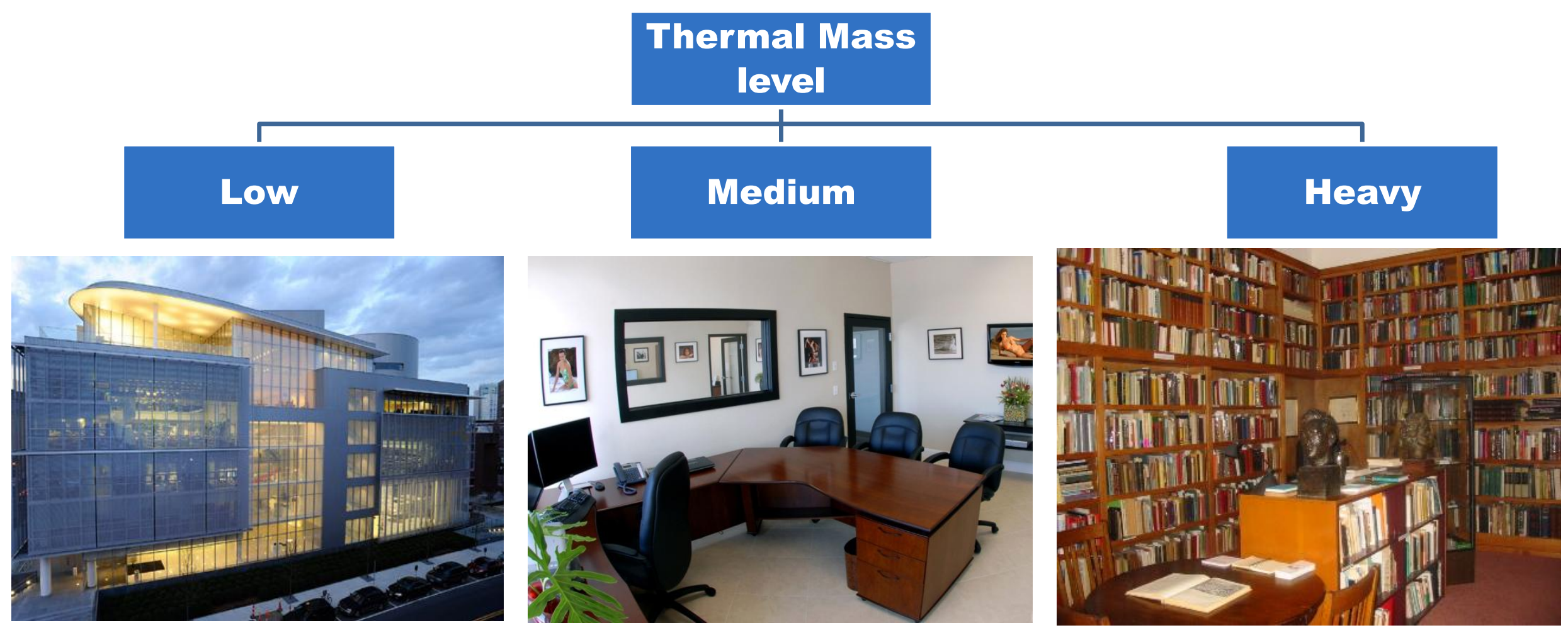




\section{Simulation and Field Test of 11 Buildings}
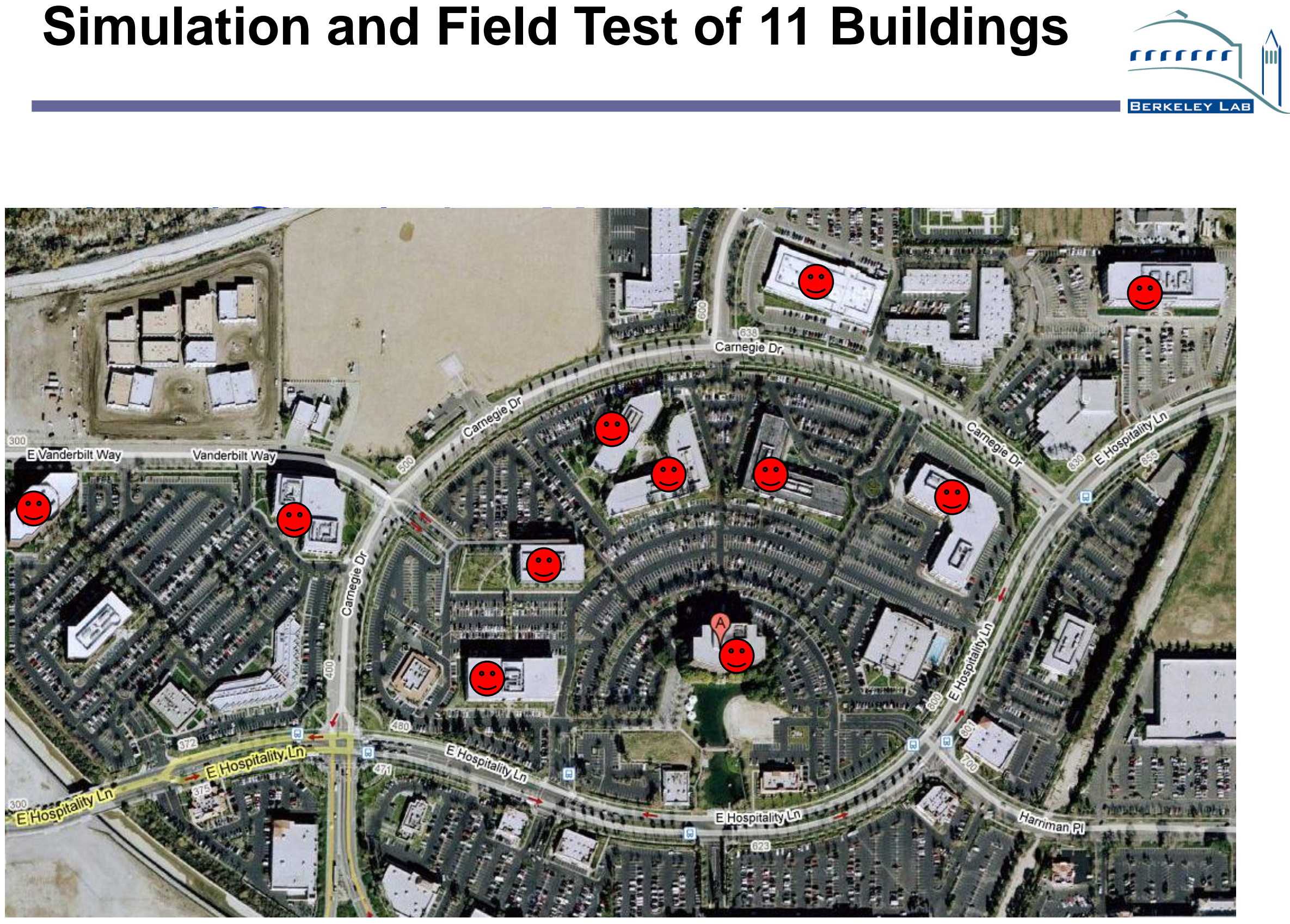


\section{Building Construction Inputs}

\begin{tabular}{|c|c|c|c|c|c|c|c|c|}
\hline \multicolumn{9}{|c|}{ BUILDING BASIC INPUT - INITIAL VALUE } \\
\hline SITE NO. & SITE NAME & $\begin{array}{l}\text { GROSS } \\
\text { (SQ FT) }\end{array}$ & $\begin{array}{l}\text { LENGTH } \\
\text { (FT) }\end{array}$ & $\begin{array}{l}\text { WIDTH } \\
\text { (FT) }\end{array}$ & $\begin{array}{c}\text { FLOOR } \\
\text { HEIGHT } \\
\text { (FT) }\end{array}$ & WWR_SN & WWR_EW & $\begin{array}{c}\text { BUILDING } \\
\text { ORIENTATION }\end{array}$ \\
\hline$\# 1$ & Two Carnegie Plaza & 68,955 & 300 & 115 & 12 & 0.50 & 0.50 & 45 \\
\hline$\# 2$ & One Carnegie Plaza-A & 62,800 & 300 & 105 & 12 & 0.50 & 0.50 & 315 \\
\hline \#3 & One Carnegie Plaza -B & 38,808 & 270 & 70 & 12 & 0.50 & 0.50 & 45 \\
\hline$\# 4$ & One Vanderbilt & 73,730 & 205 & 90 & 12 & 0.25 & 0.25 & 315 \\
\hline \#5 & One Parkside & 70,069 & 175 & 100 & 12 & 0.60 & 0.60 & 0 \\
\hline \#6 & Lakeside Tower & 112,717 & 210 & 90 & 12 & 0.60 & 0.60 & 0 \\
\hline$\# 7$ & Two Parkside & 80,750 & 250 & 110 & 12 & 0.40 & 0.40 & 0 \\
\hline$\# 8$ & Three Carnegie Plaza & 83,698 & 420 & 100 & 12 & 0.40 & 0.40 & 45 \\
\hline$\# 9$ & Brier Corporate Center & 104,501 & 350 & 100 & 12 & 0.40 & 0.40 & 45 \\
\hline \#10 & Vanderbilt Plaza & 119,035 & 200 & 150 & 12 & 0.40 & 0.40 & 0 \\
\hline \#11 & Inland Regional Center & 81,079 & 350 & 115 & 12 & 0.30 & 0.30 & 45 \\
\hline
\end{tabular}




\section{Initial Model Parameters}

- Internal loads densities

- Occupancy - 390 sqft per person

- Lighting-Estimated from building type and year built, using Title 24

- $1.8 \mathrm{~W} / \mathrm{sqft}$ for oldest and $1.2 \mathrm{~W} / \mathrm{sqft}$ for newest

- Plug load-0.75 W/sqft

- Internal load schedules were set to match actual building operating hours

- Interior mass was set to medium unless otherwise specified in owner surveys 


\section{Internal Load Schedules}

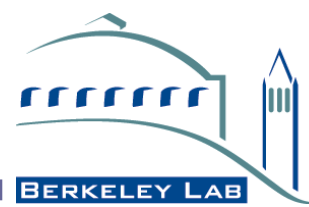

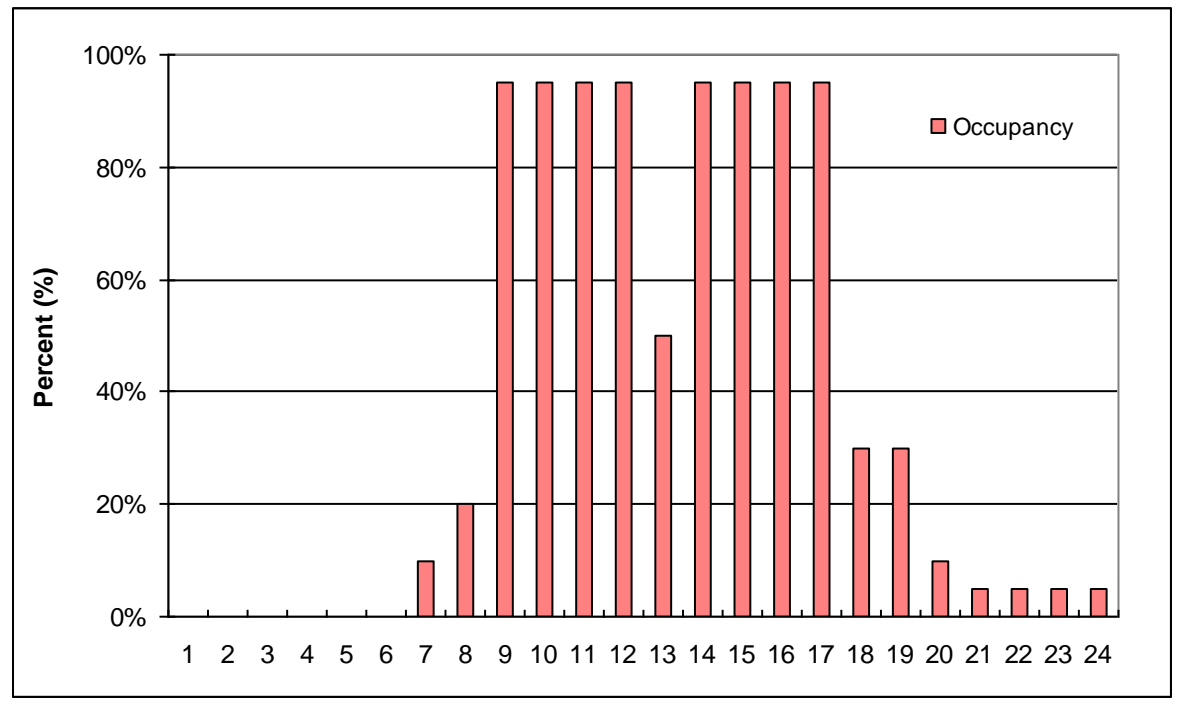

Occupancy

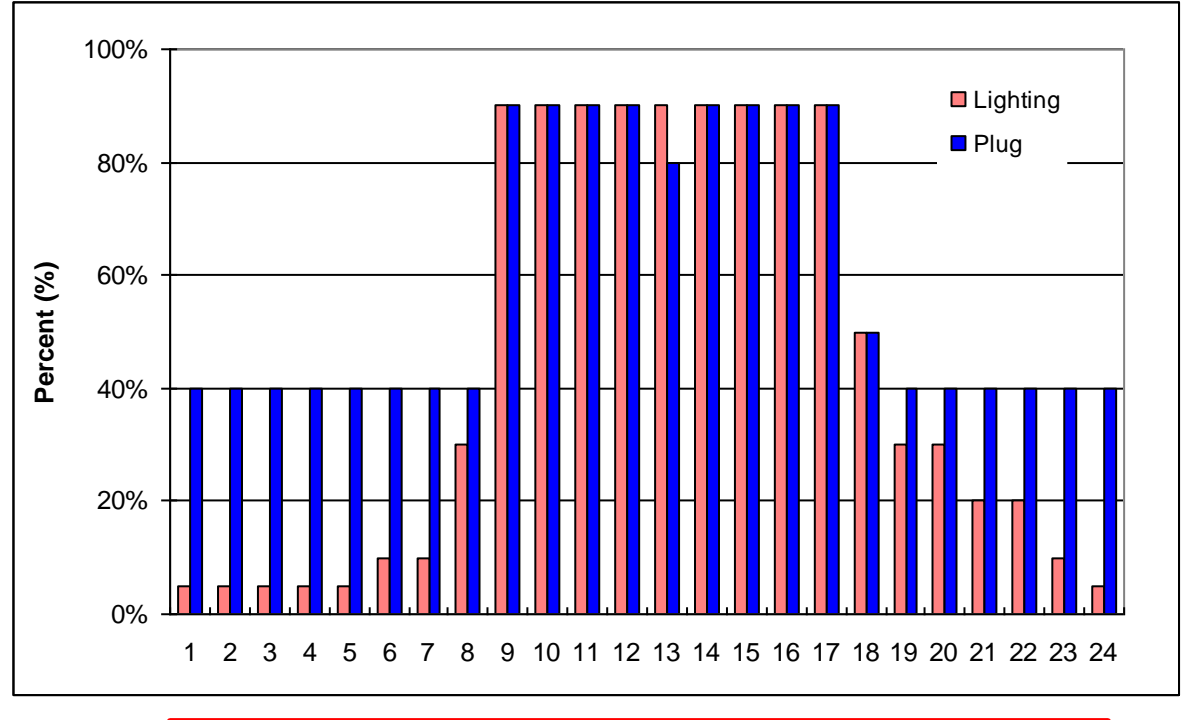

Lighting and Equipment 


\section{Initial HVAC System Parameters}

- Water-Cooled (Central chiller) and Air-Cooled (Package DX Units)

- Air Distribution Type - VAV

- Normal Zone Temperature Set Points - $77^{\circ} \mathrm{F}$

- Operating Schedules - Site survey or demand profile (measured data) 


\section{Initial Simulation Results}

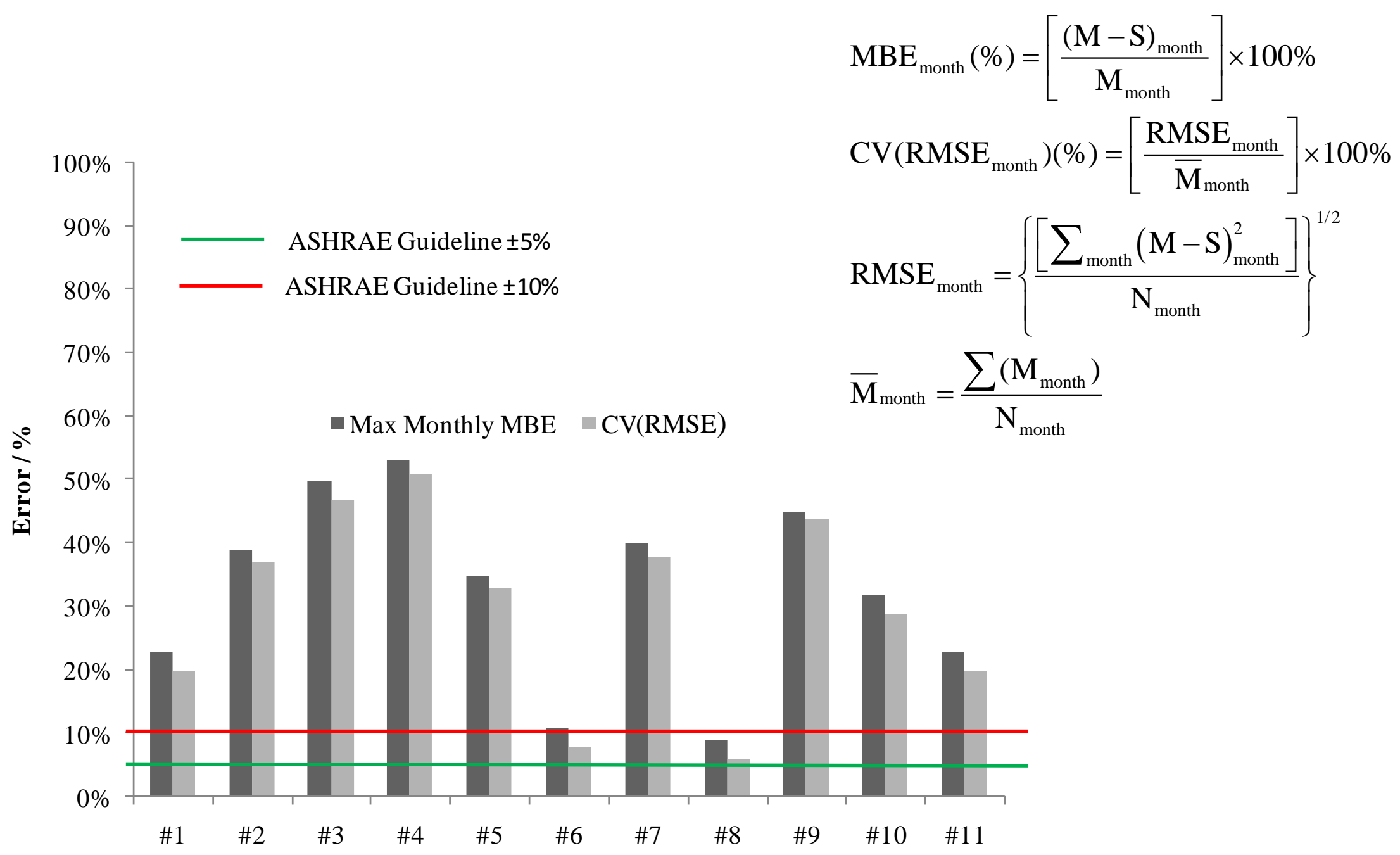




\section{Simulation Model Adjustments}

- Weather data - Replace the TMY weather file with actual weather data measured at or very near the buildings

- Internal loads - Adjust lighting and equipment densities and operating schedules

- Use the whole building electrical data to estimate the magnitudes of the internal densities and shapes of the operating schedules

- Schedule any special circumstances in to the model. In this case, he cooling plants were locked out when the outside temperature was lower than $55^{\circ} \mathrm{F}$ so that schedule was added to the model 


\section{Internal load adjustments}

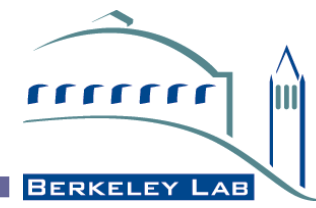

\begin{tabular}{|lcccc|}
\hline \multirow{2}{*}{ Site No. } & Year Constructed & $\begin{array}{c}\text { Lighting Density } \\
\text { (w/sq ft) }\end{array}$ & \multicolumn{2}{c|}{ Plug Load Density (w/sq ft) } \\
\cline { 4 - 5 } & 1990 & 1.60 & Initial & After calibration \\
\hline$\# 1$ & 1988 & 1.60 & 0.75 & 0.75 \\
\hline$\# 2$ & 1988 & 1.60 & 0.75 & 1.50 \\
\hline$\# 3$ & 1988 & 1.60 & 0.75 & 1.50 \\
\hline$\# 4$ & 1993 & 1.60 & 0.75 & 1.80 \\
\hline$\# 6$ & 1990 & 1.60 & 0.75 & 1.40 \\
\hline$\# 7$ & 2001 & 1.20 & 0.75 & 0.90 \\
\hline$\# 8$ & 2003 & 1.20 & 0.75 & 1.50 \\
\hline$\# 9$ & 2005 & 1.10 & 0.75 & 0.60 \\
\hline$\# 10$ & 2002 & 1.20 & 0.75 & 1.40 \\
\hline$\# 11$ & 1994 & 1.60 & 0.75 & 1.00 \\
\hline
\end{tabular}




\section{Adjusted internal load schedules for BIdg. 8}
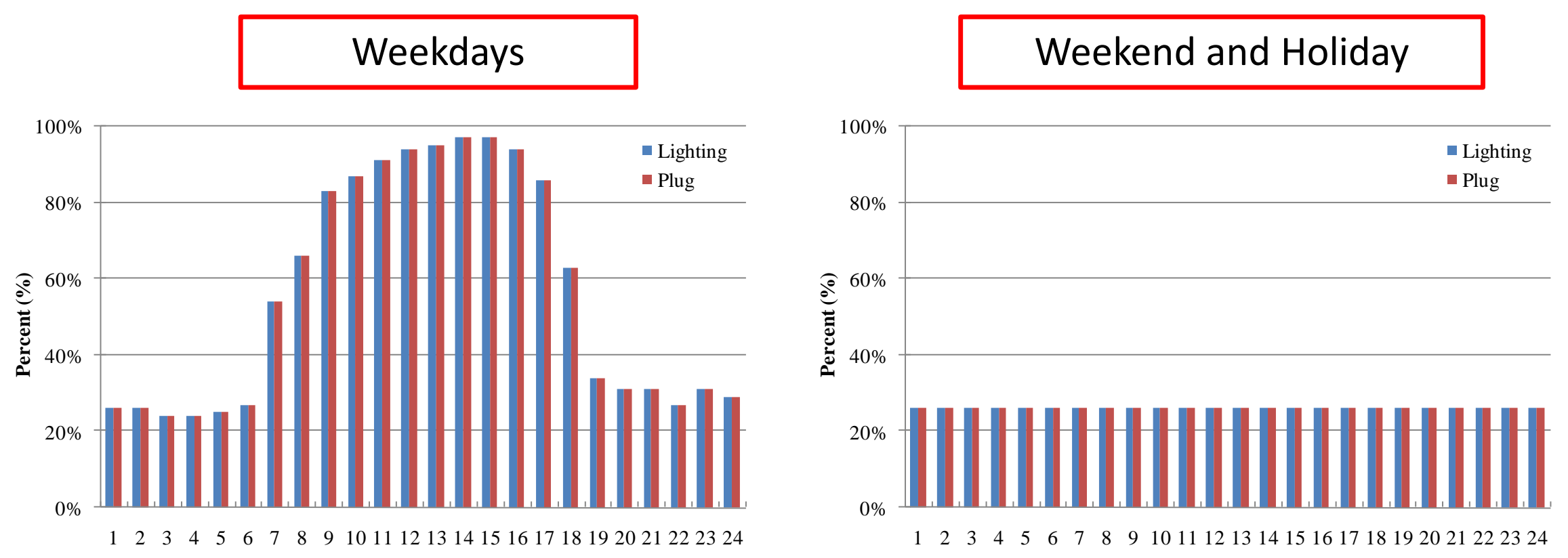


\section{Adjusted Model Performance-Monthly}

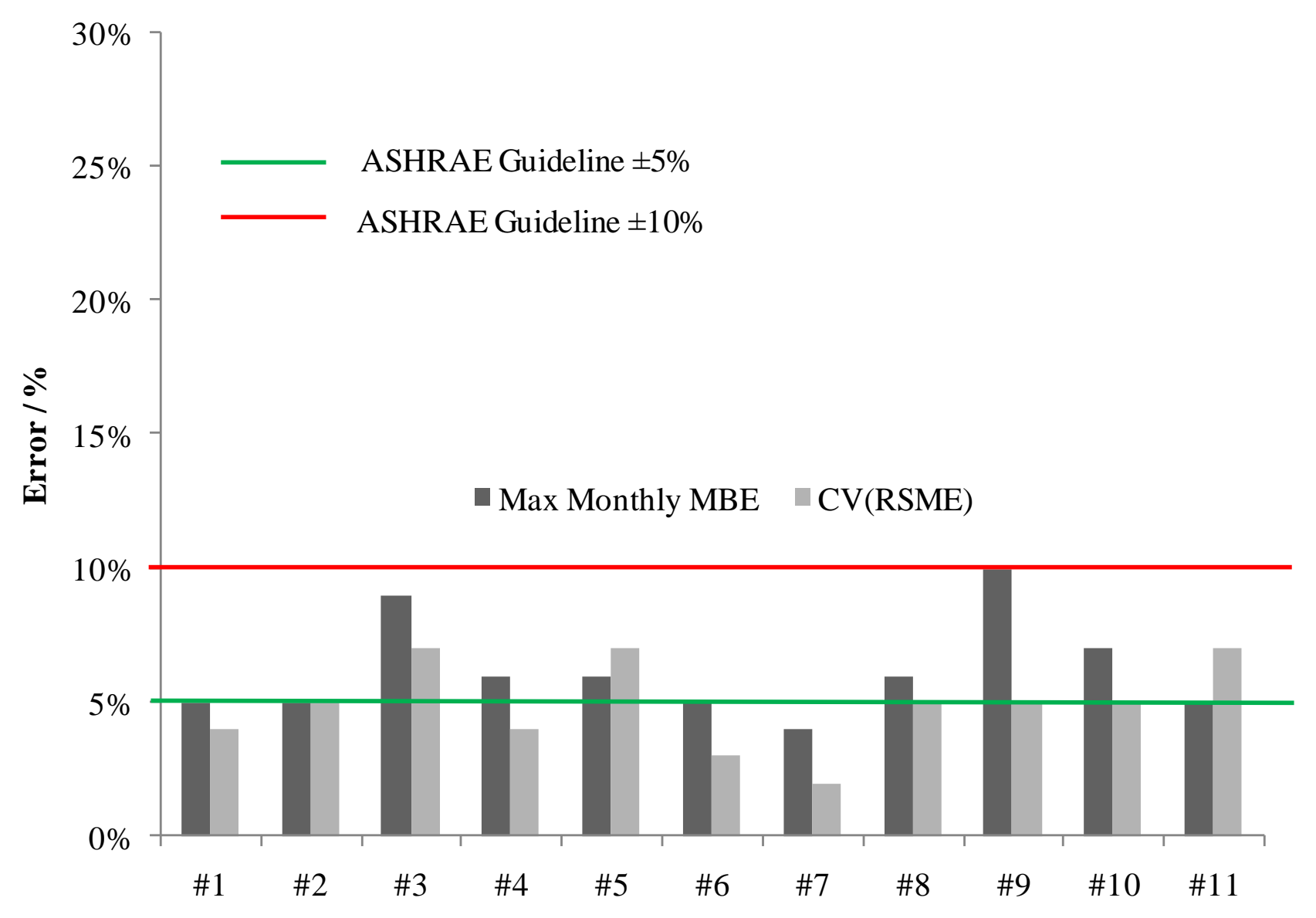




\section{Adjusted Model Performance-Daily}

- Measured Electrical Demand $\pm 15 \%$ (Aug.20 to 24)

— Simulated Electrical Demand (Aug.20 to 24)

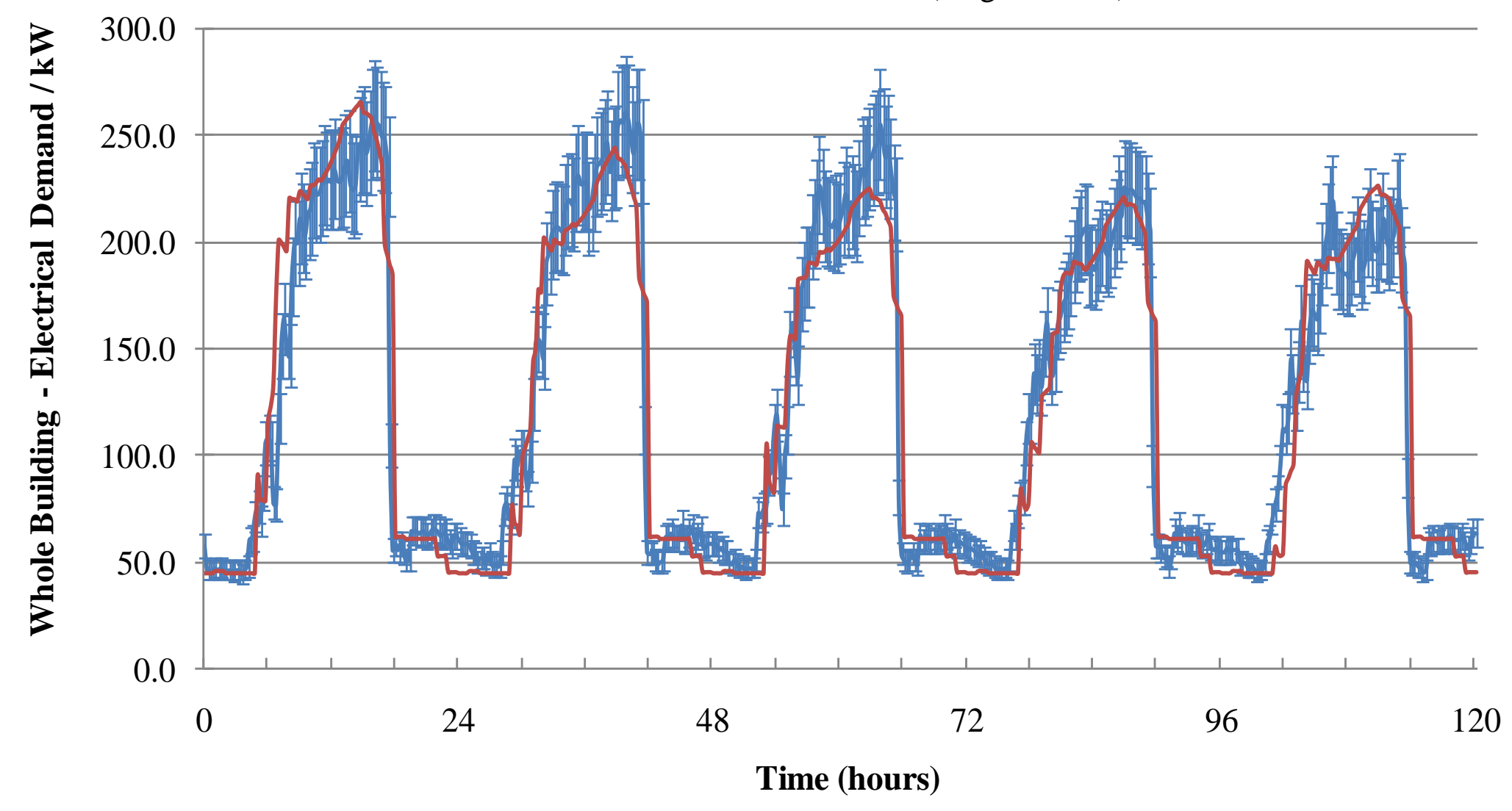




\section{Pre-cooling Strategies}

$\leadsto$ Baseline

- Pre-cooling with exponential temp reset

$\leftarrow$-Pre-cooling with step temp reset
- - Pre-cooling with linear temp reset

$*$ No pre-cooling with exponential temp reset

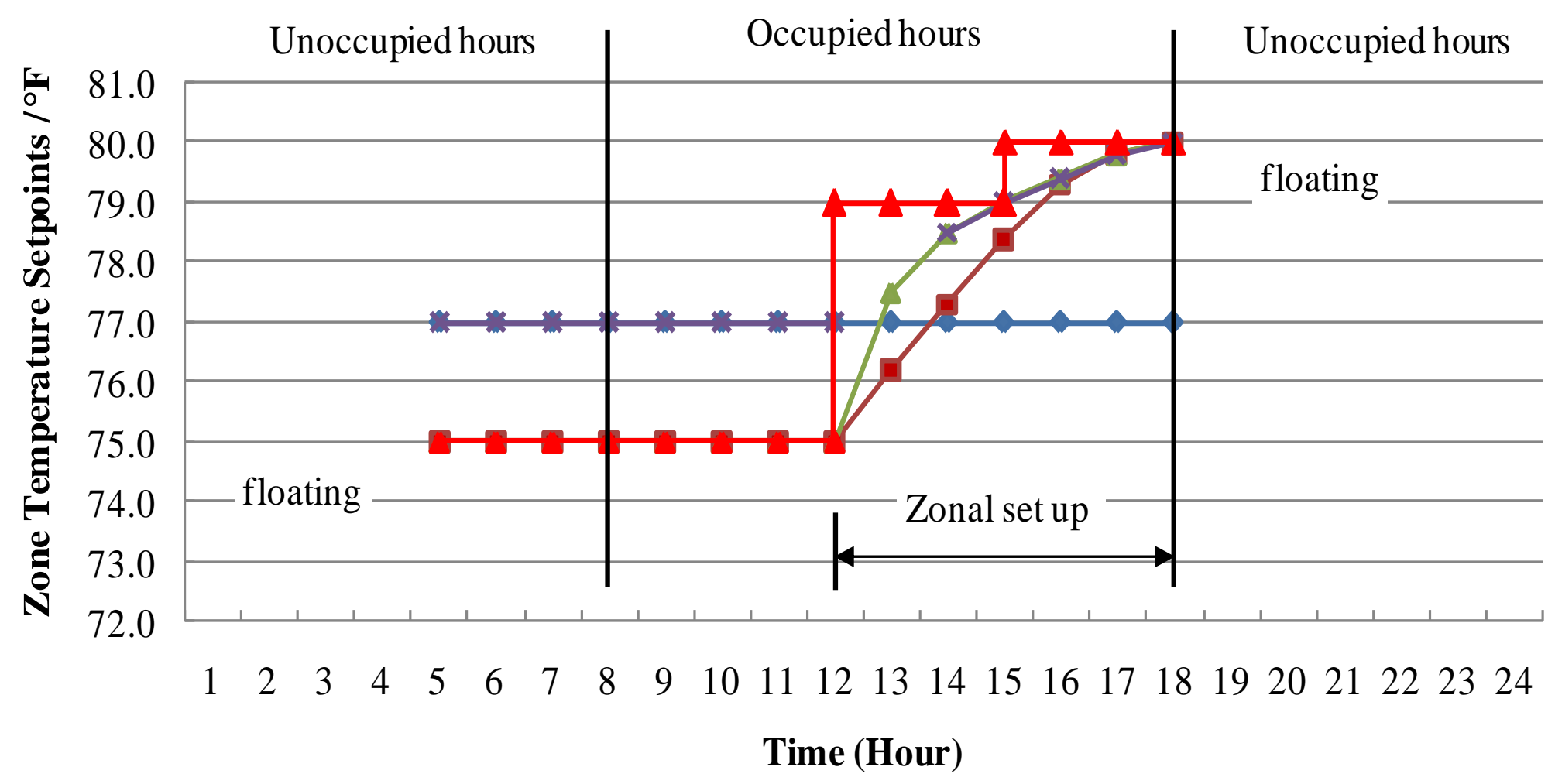




\section{Simulations of Pre-cooling Strategies}

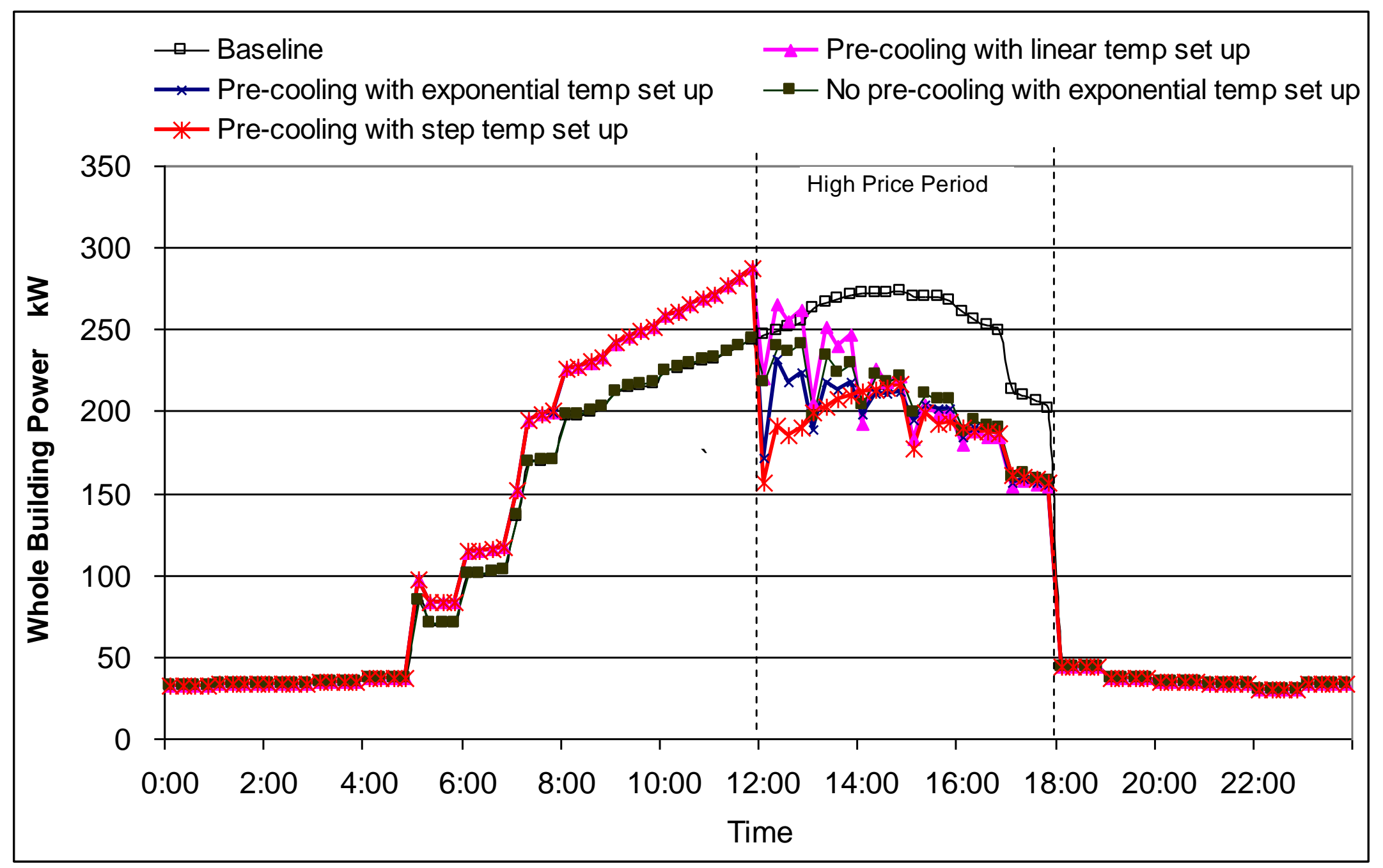




\section{Measured Pre-cooling Implementation}

Building site \# 8, 9/3/2008 (Max OAT: $36.7^{\circ} \mathrm{C}$ )

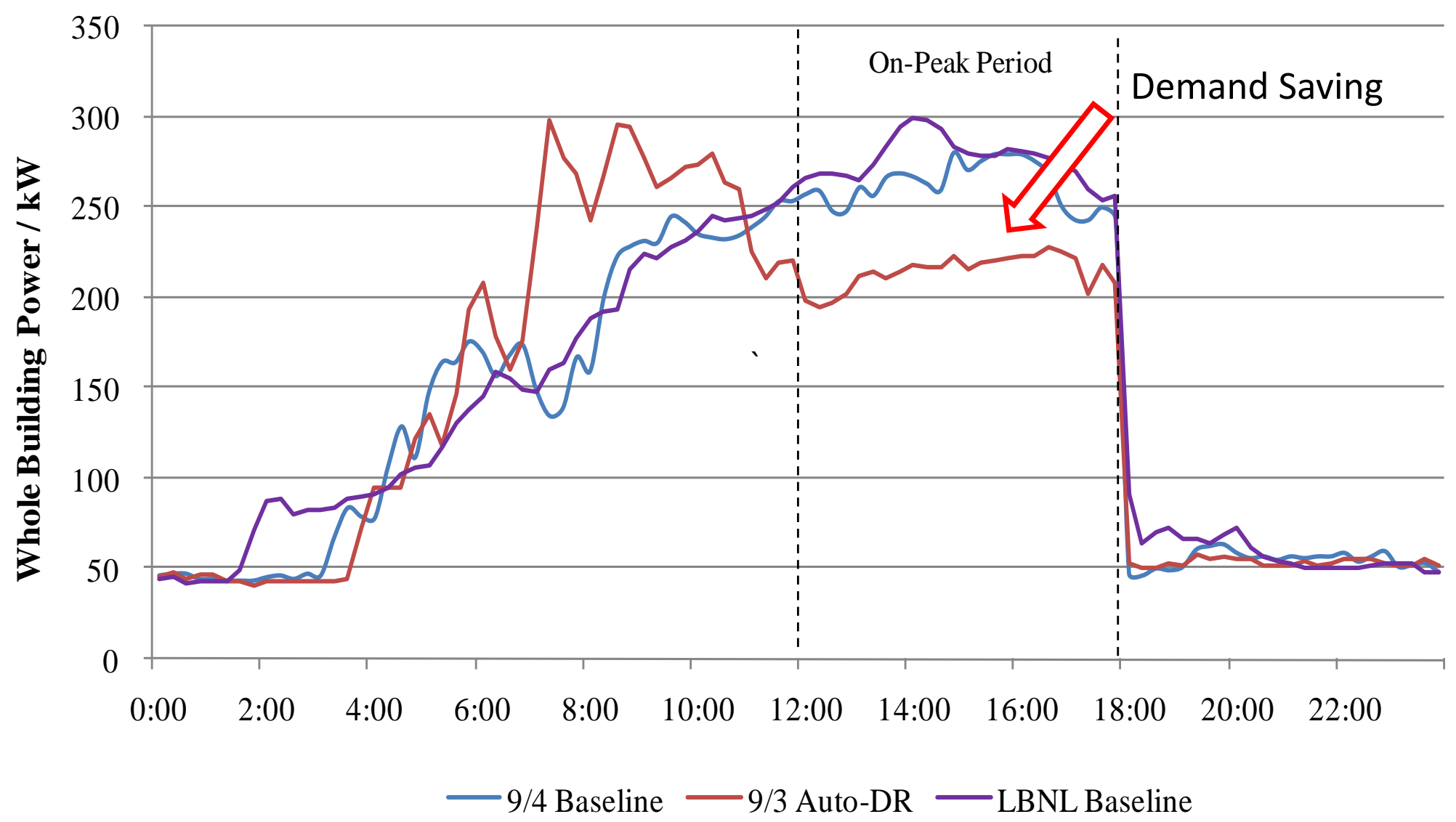




\section{Predicted vs. Measured Demand Savings}

- Actual data $\quad$ Simulation Results

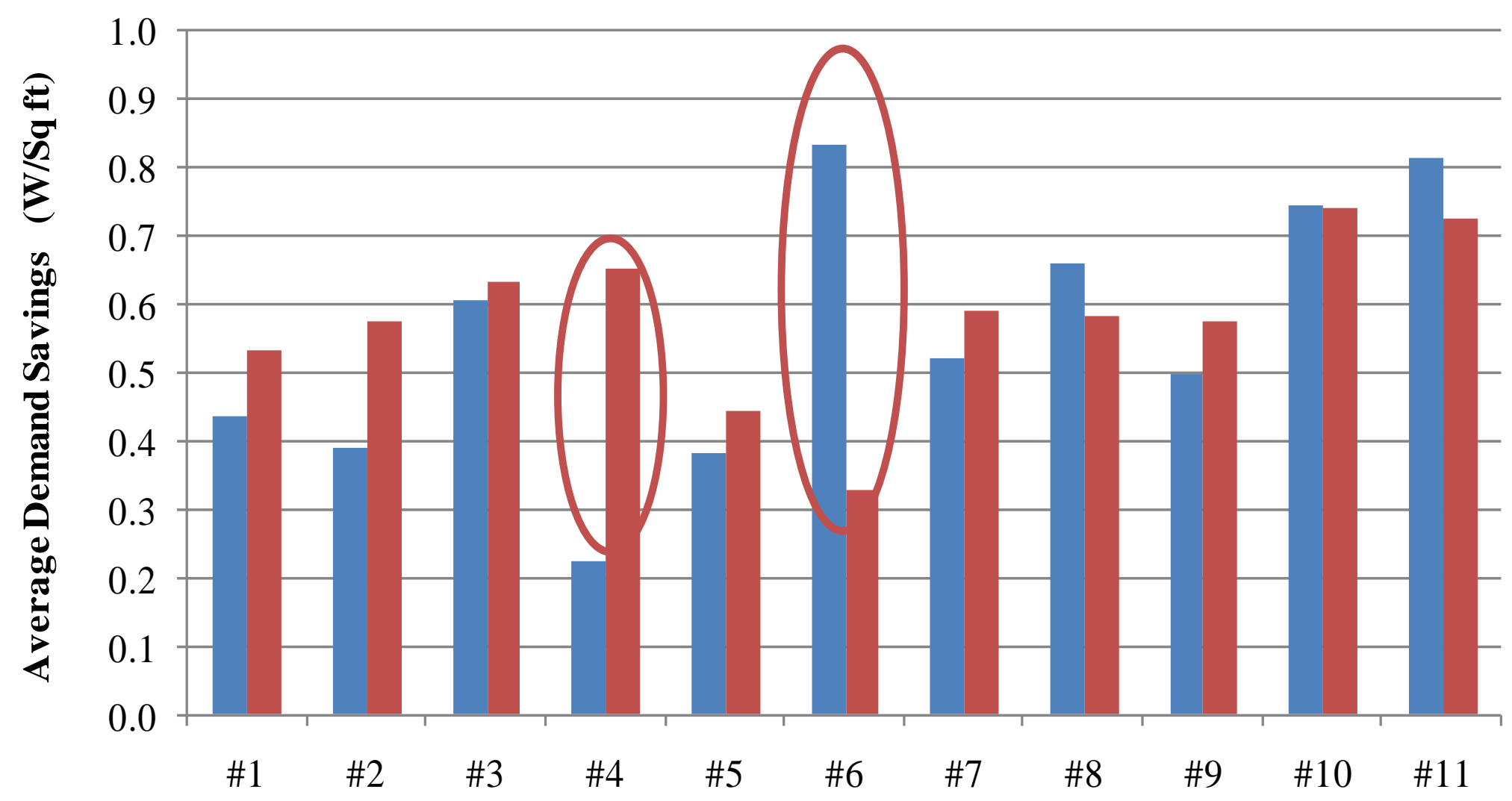




\section{Pre-cooling Field Test Analysis}

Discrepancy between Measured and Simulated Demand Savings in Site \#6

Building site \# 6, 8/12/2008 (Max OAT: $95{ }^{\circ}$ F)

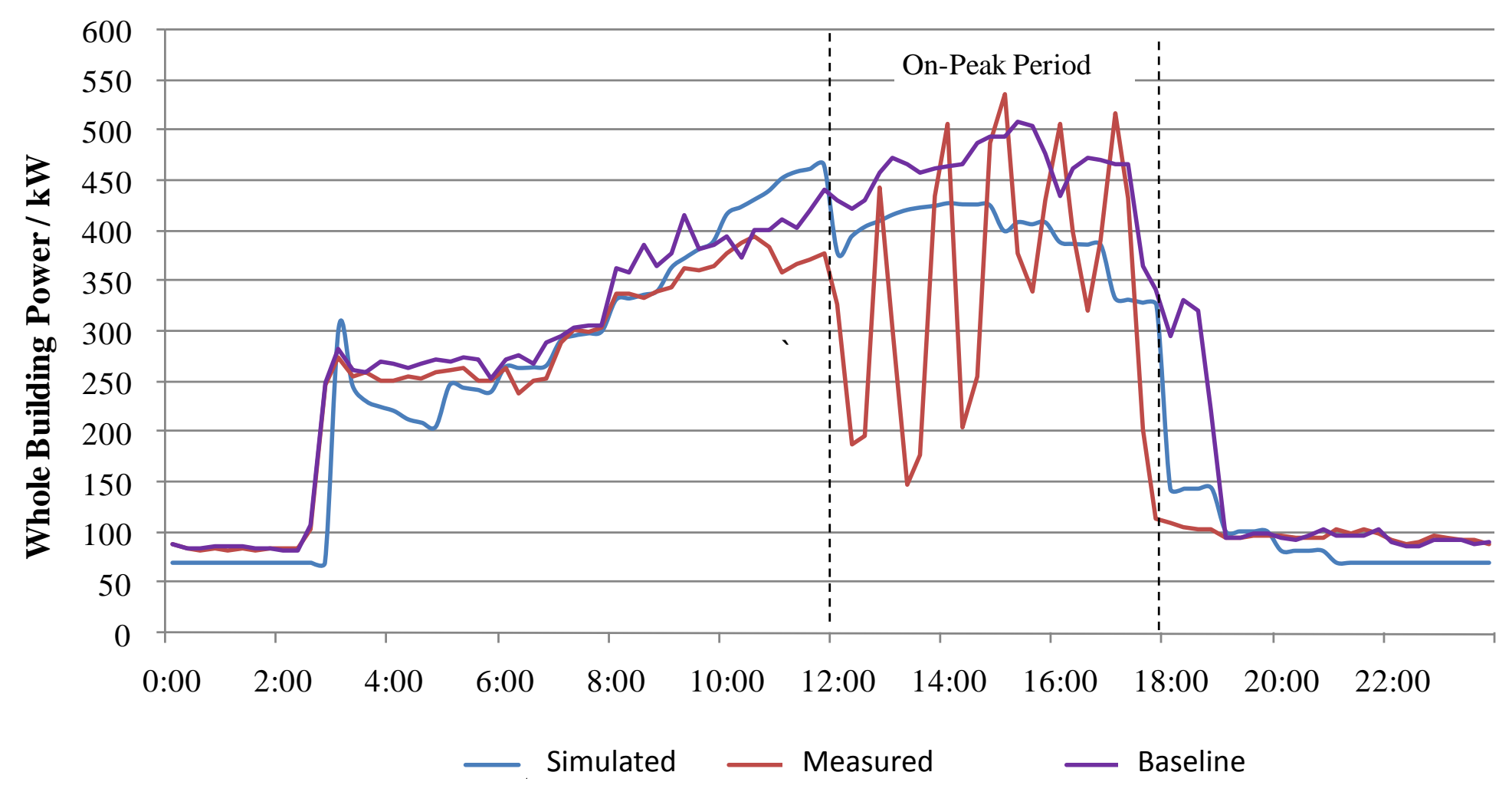




\section{DRQAT Model Adjustments in Two Steps}

1a. Use actual weather data

1b. Model input adjustments

- Building information

- Lighting \& plug load power densities, operating schedules

- Occupant number, operating schedules

- HVAC system characteristics

- Plant equipment characteristics

2. Model input adjustments based on Step 1 model outputs

- Using DRQAT zone temperature output check if set-points are met and maintained

- If not increase zone sizing factor, which will increase simulated cooling capacity 


\section{Winter Electric Demand to Adjust Lighting and Plug Load Density and Schedule}

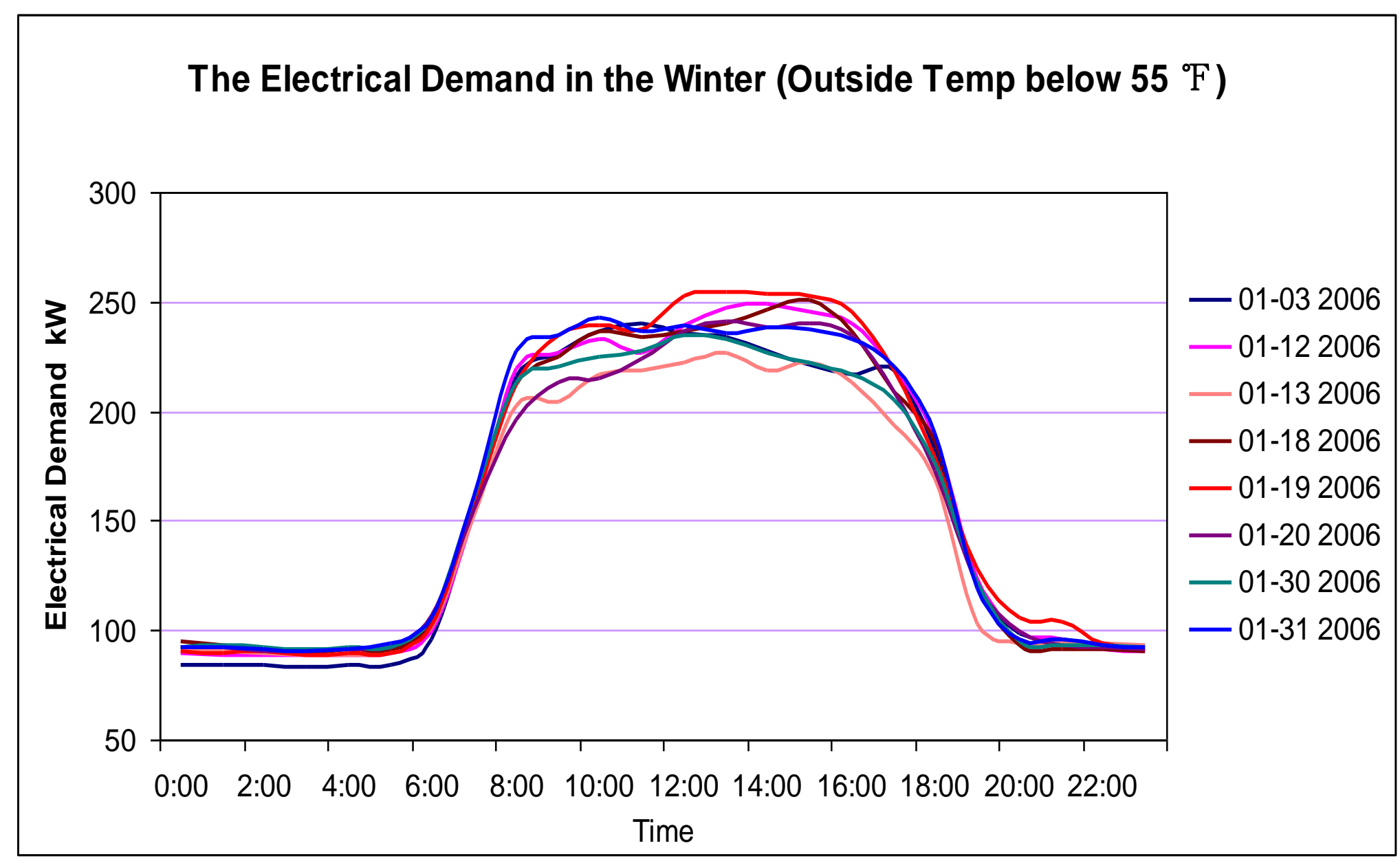




\section{Adjusted lighting \& plug load densities and schedules}

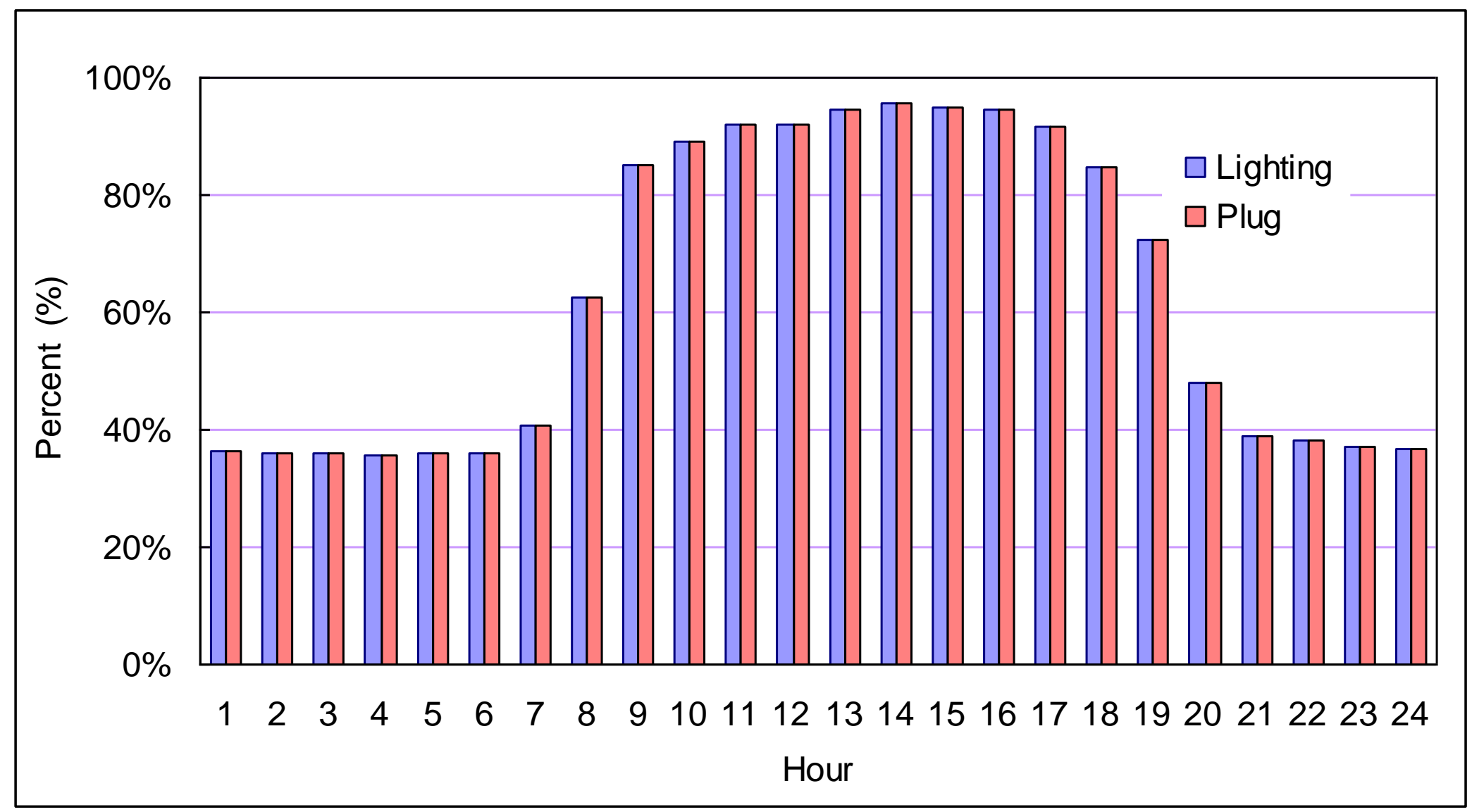




\section{Compare Step 1 Adjusted Model to Measured Loads}
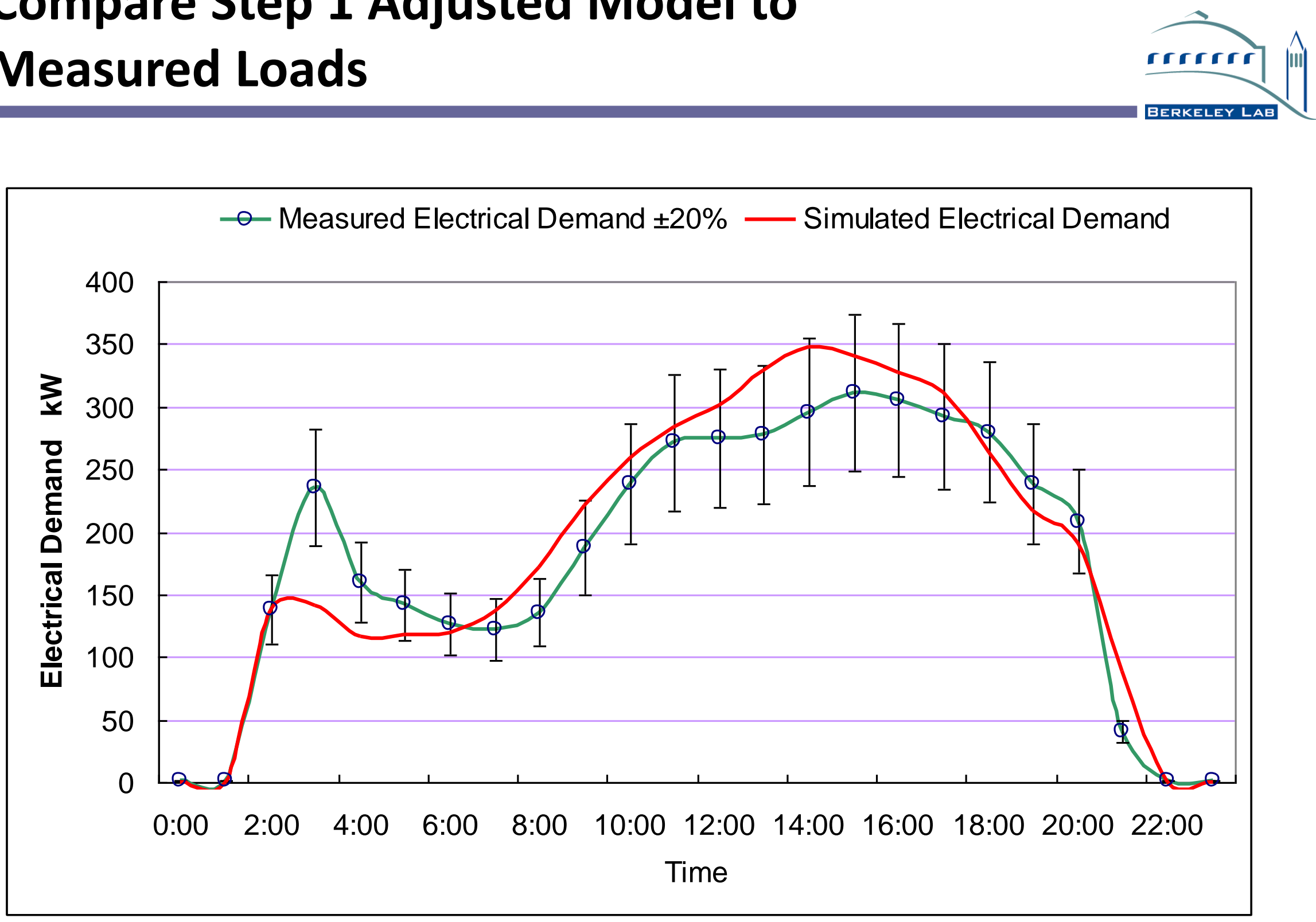


\section{Compare DRQAT Step 1 Model Monthly Energy Consumption Predictions to Measured Data}

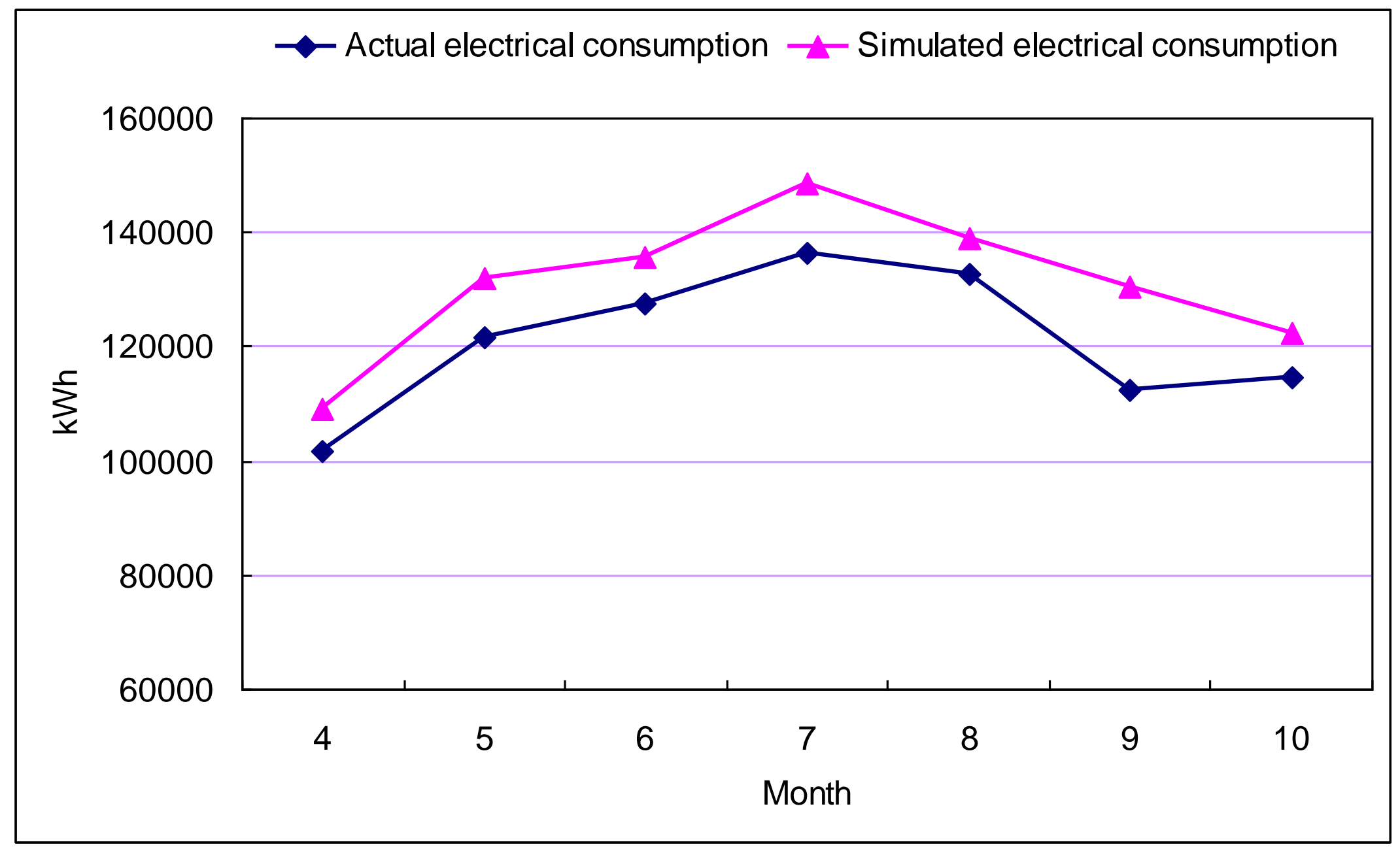




\section{DRQAT Model Adjustments-Step 2}

\section{1a. Use actual weather data}

1b. Model input adjustments

- Building information

- Lighting \& plug load power densities, operating schedules

- Occupant number, operating schedules

- HVAC system characteristics

- Plant equipment characteristics

2. Model input adjustments based on Step 1 model outputs

- Using DRQAT zone temperature output check if set-points are met and maintained

- If not increase zone sizing factor, which will increase simulated cooling capacity 
Simulated zone temperatures unable to reach set-point 


\section{Compare DRQAT Step 2 Model Monthly Energy Consumption Predictions to Measured Data}

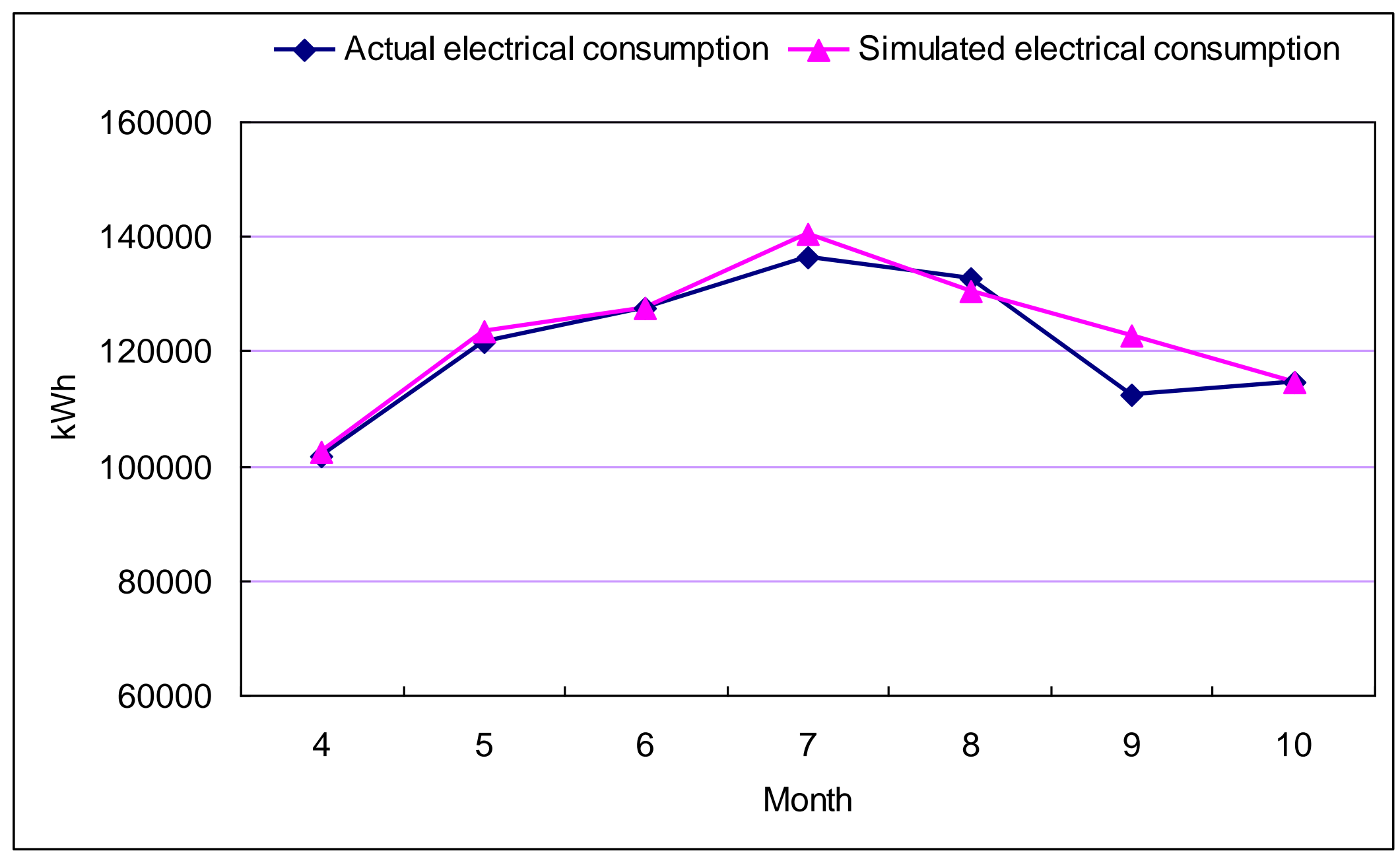




\section{Daily electrical demand comparison in May}
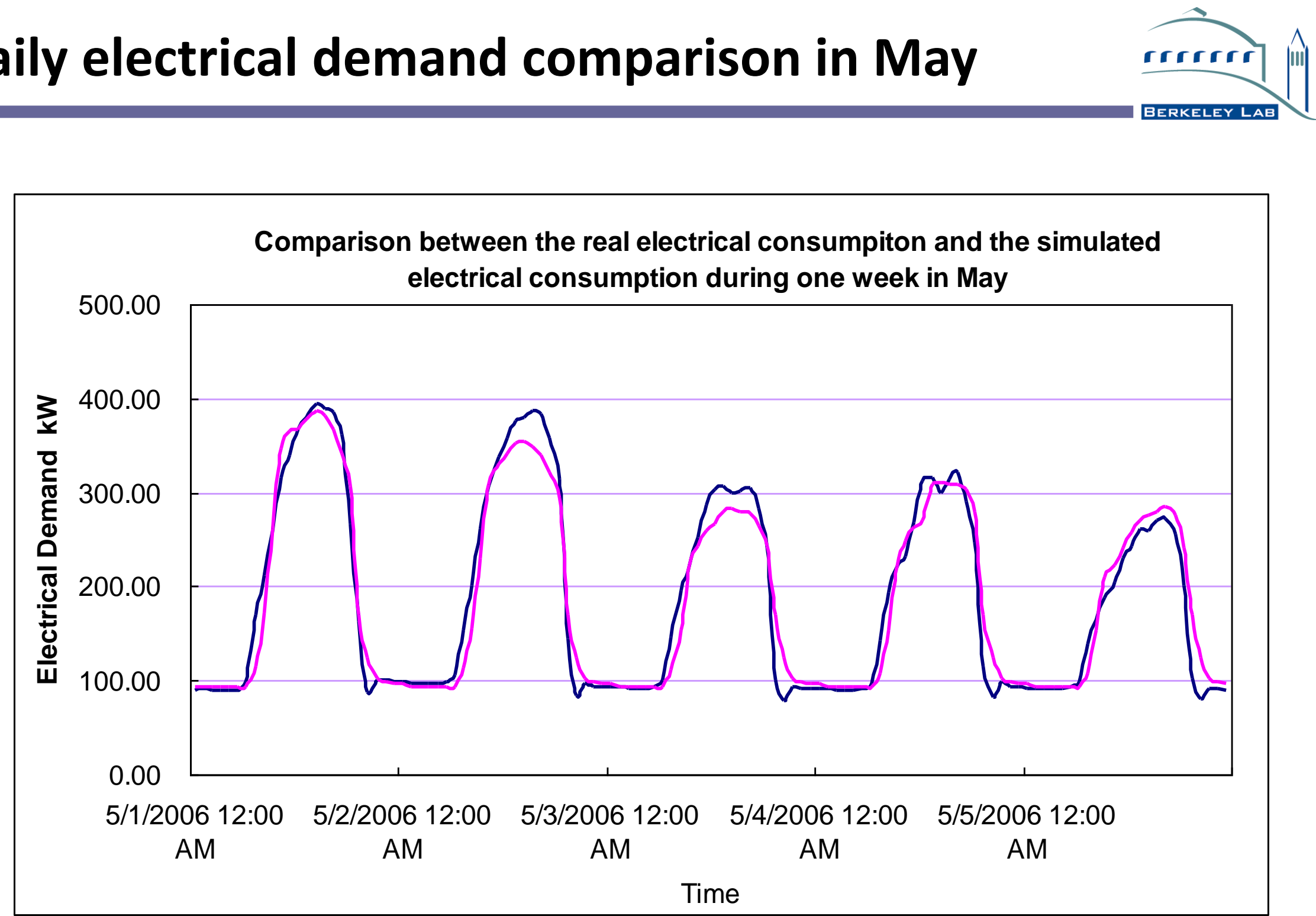


\section{Daily electrical demand comparison in June}
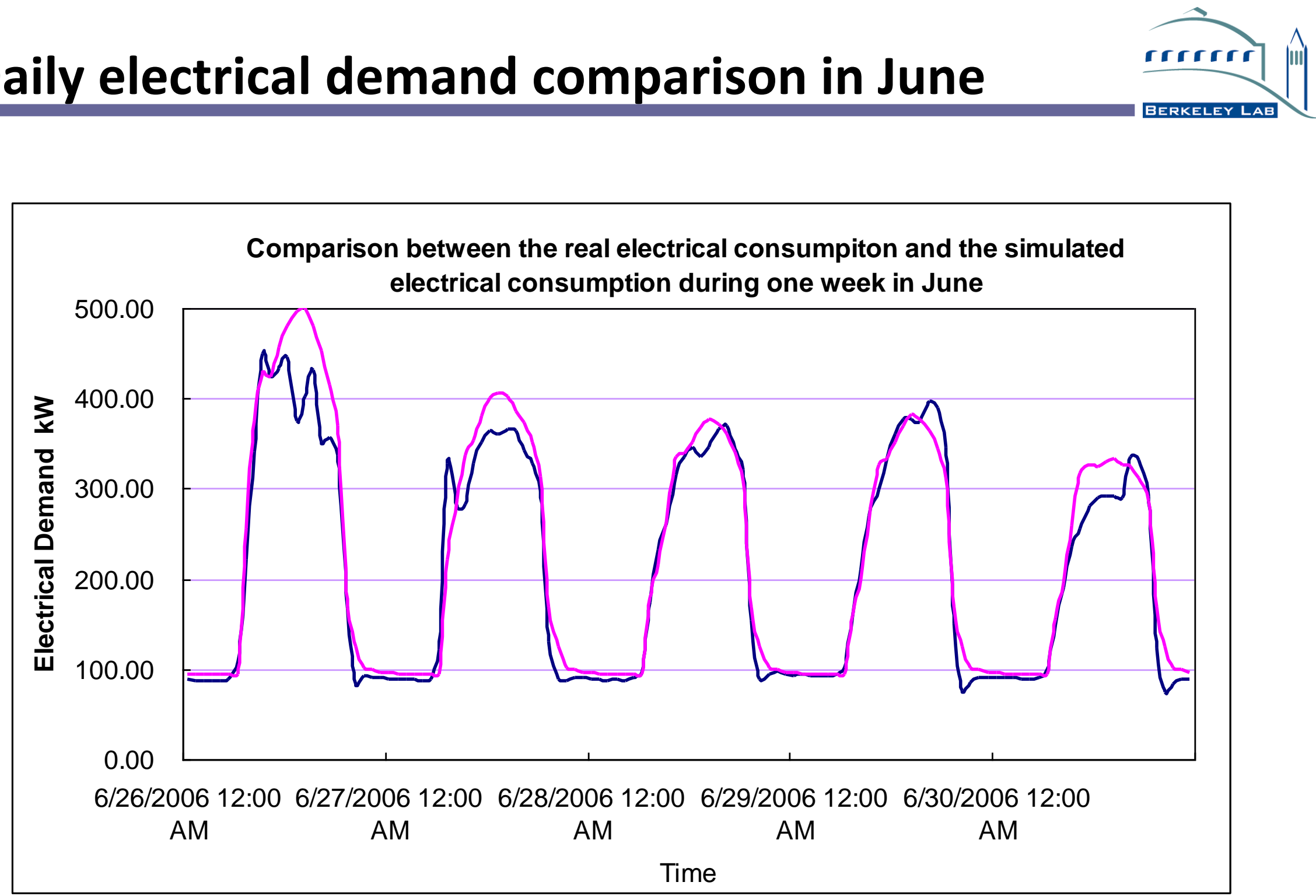


\section{Conclusions}

- Demonstrated how to optimize and verify pre-cooling strategies for the office buildings in hot climate zone with the assistance of Demand Response Quick Assessment Tool (DRQAT)

- The field test results indicated that the pre-cooling strategies were able to reduce the peak demand as expected on Auto-DR test days (15 30\% throughout the summer period)

- The demand shed predicted by the adjusted DRQAT models match well with the actual data on Auto-DR days when HVAC equipment were operated properly 


\section{Lessons Learned}

- Use real weather data - Outside air sensor or weather station installed at the building

- Use sub-metered HVAC and whole building demand data to adjust model inputs - direct measurement or short-term measurement

- Estimate more accurate internal mass level based on building constructions

- Simulation cannot simulate improper equipment and operation

- Lighting and plug load densities and schedules can be estimated from winter electric demand data when electricity is not used for heating

- Simulated zone temperatures that cannot meet or maintain the setpoint value indicate that the sizing factor should be increased 


\section{Using DRQAT for Parametric Studies}

- Background

- "Pre-cooling" techniques reduce the building mass temperatures and interior temperatures in the early part of the day and reduce cooling electricity demand and electricity use in the afternoon.

- Demand response quick assessment tool - DRQAT - was developed to evaluate DR strategies in commercial buildings based on EnergyPlus models of prototypical buildings

- Objective

- Evaluate impact of building size, thermal mess level, weather, and DR strategies on peak demand savings in commercial buildings

- Study the impact of three DR control strategies: linear, step and exponential temperature reset during the peak hours 


\section{Previous Field Studies}

- Demand shed in field studies: different building sizes, DR control strategies, weather conditions...

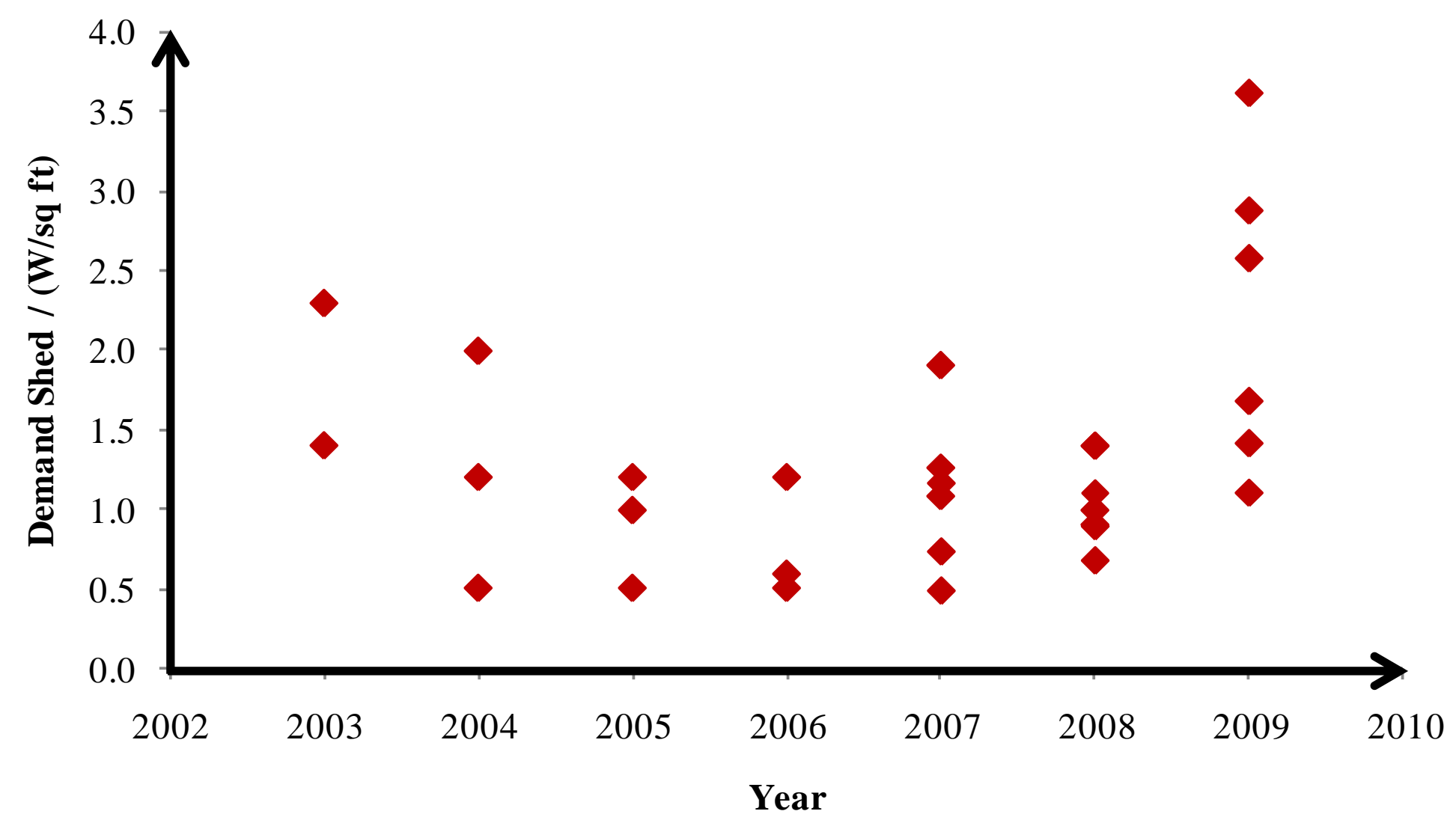




\section{Scenario Analysis}

- Weather: warm, hot, extremely hot

- Building Size: small, medium, large

- Thermal Mass: light, medium, heavy

- DR Strategy (zone temperature reset): linear, step, exponential

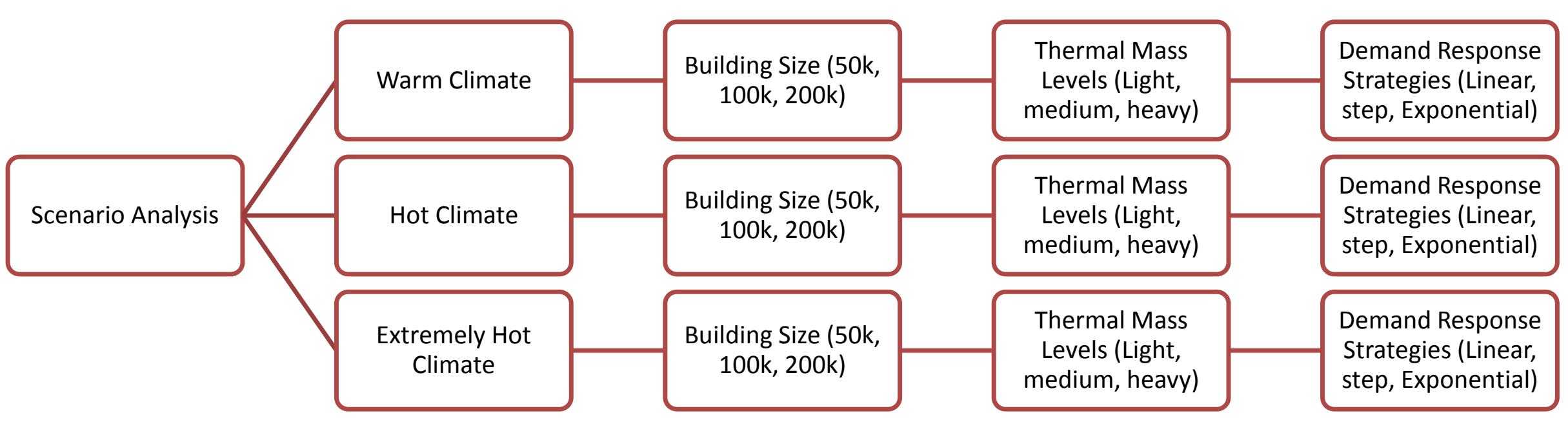




\section{Results - Effects of Thermal Mass}

- Magnitude of DR demand savings increases with building thermal mass

- Employing the same strategy - "pre-cooling with exponential temp reset" in a medium-sized concrete building

Demand Savings for Concrete Building in Climate 10

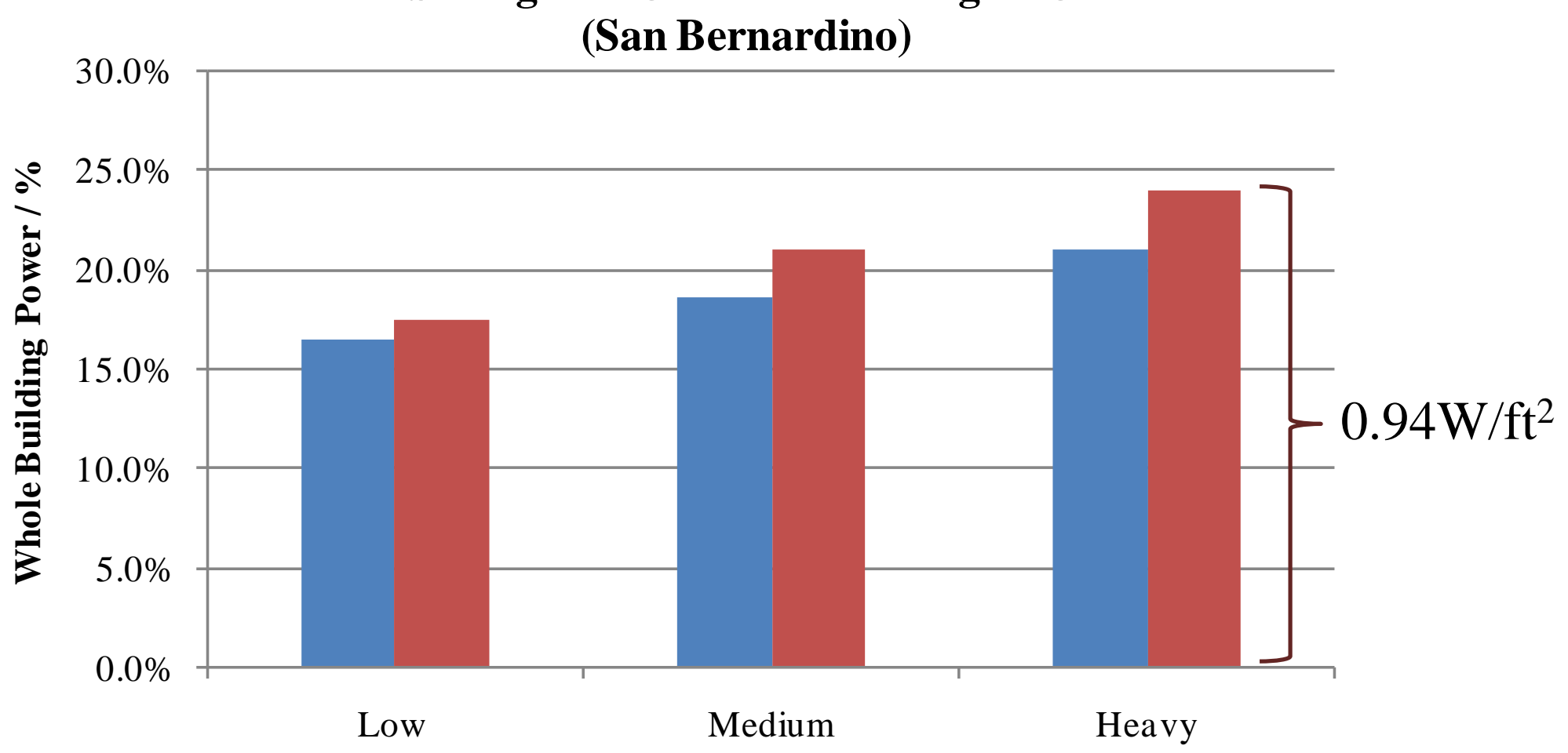

- Peak Demand Savings (12 PM - 3 PM) घeak Demand Savings (3 PM - 6 PM) 


\section{Results - Effects of Climates}

- Magnitude of DR demand savings increases with outdoor temperature

- Employing the same strategy - "pre-cooling with exponential temp reset" in a medium thermal mass building

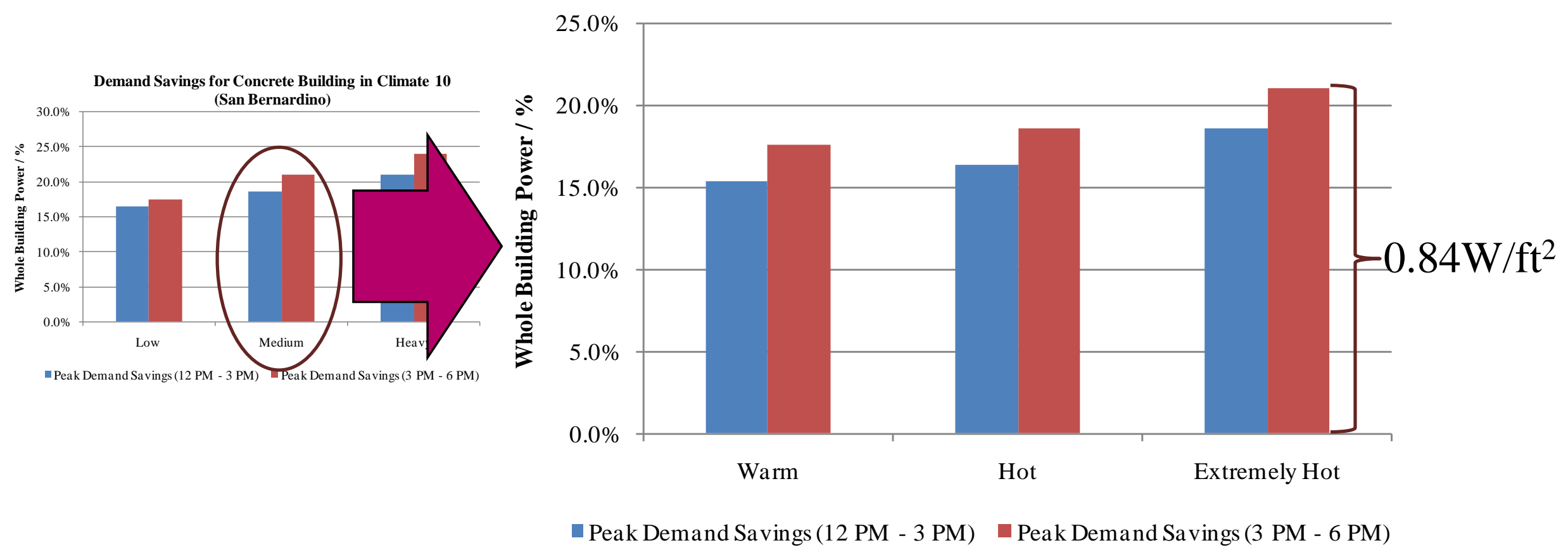




\section{DR Pre-cooling Strategies}

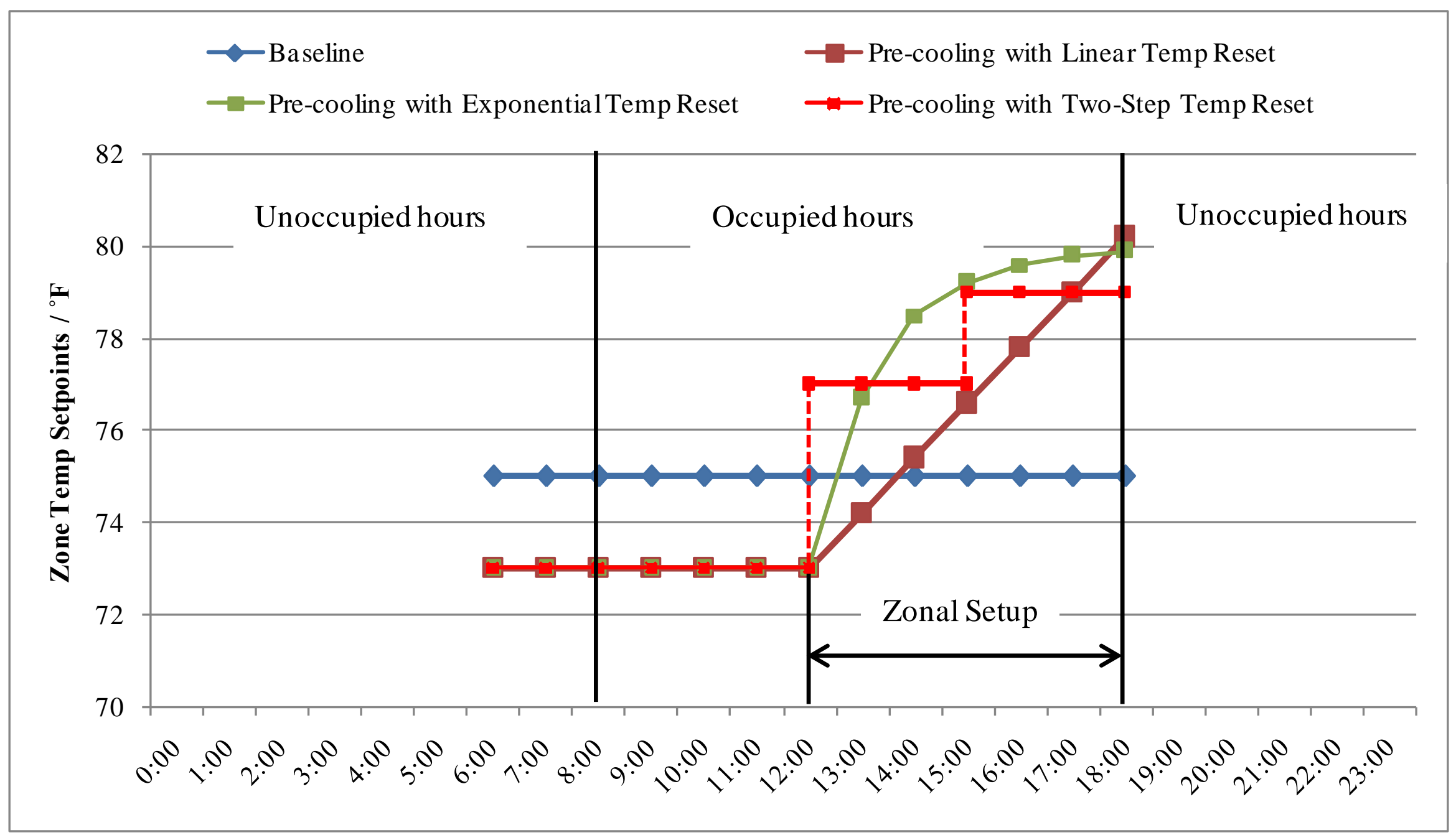




\section{Results - Effects of DR Strategies}

- The exponential temperature reset pre-cooling strategy yields the flattest demand profile during the DR event.

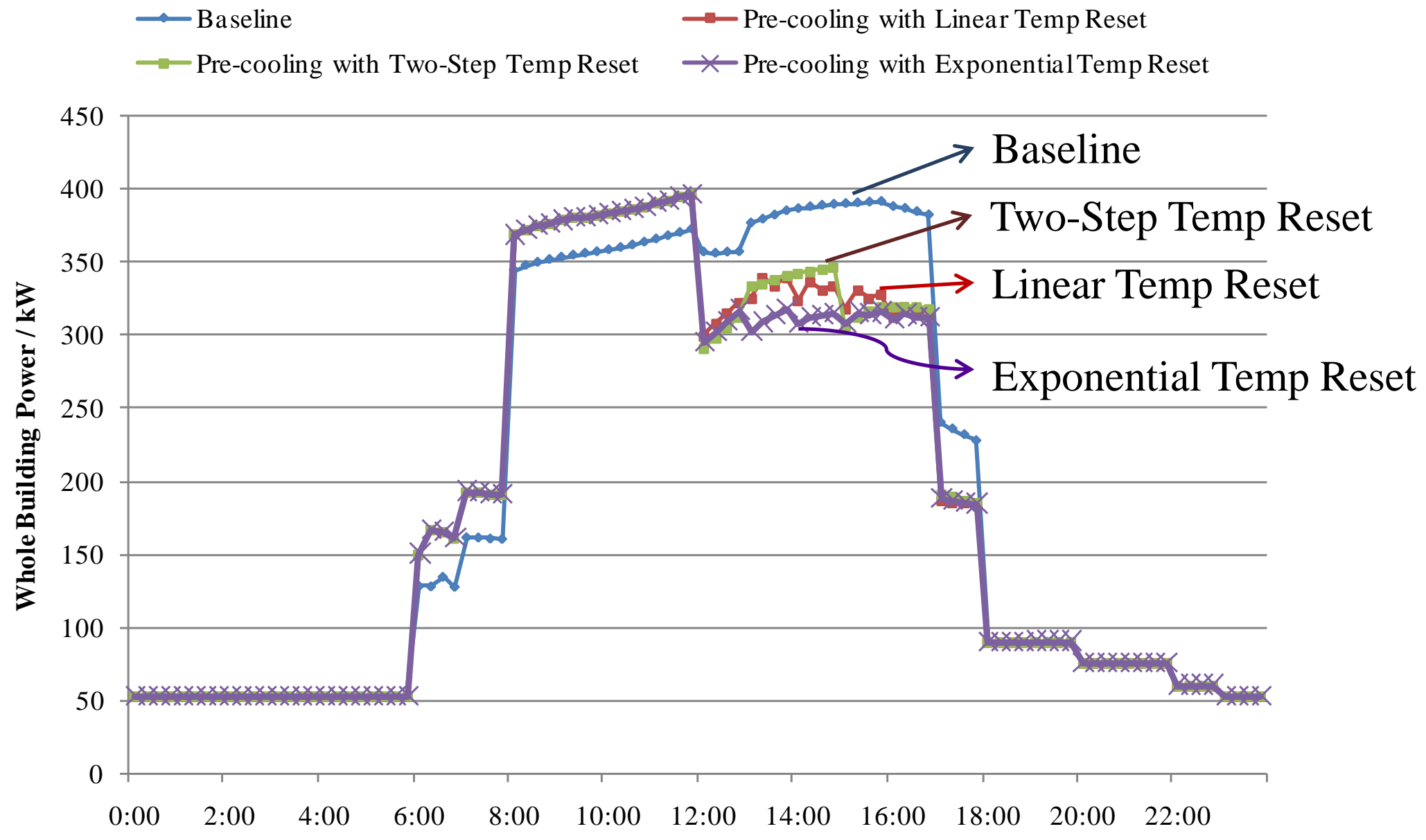




\section{Results - Zone Temperature}

- The increase in average zone temperature during DR events with "pre-cooling with exponential temp reset" is inversely related to building thermal mass

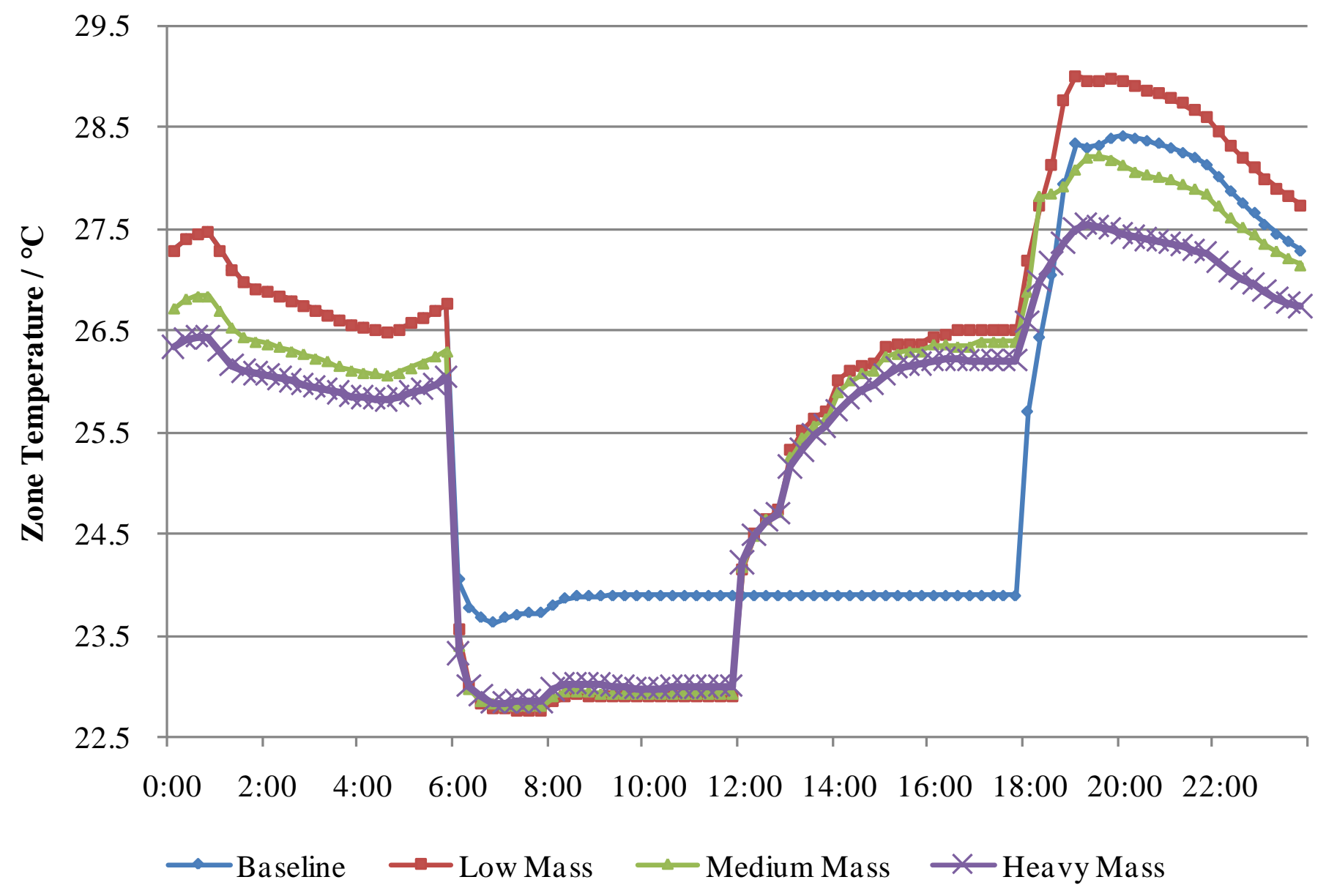




\section{Results - Zone Comfort}

- Maintaining comfort during DR events is more difficult in buildings with low thermal mass

$\multimap$ Baseline $\rightarrow$ Low Mass $\multimap$ Medium Mass $\rightleftharpoons$ High Mass

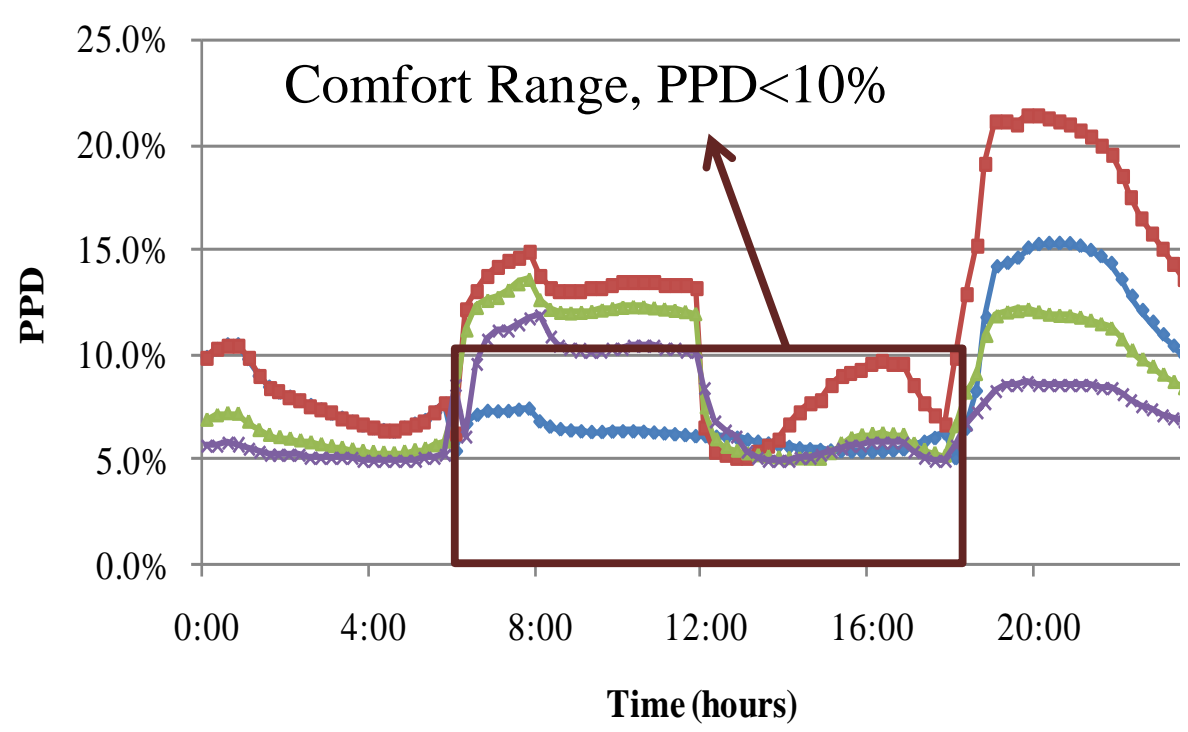

$\multimap$ Baseline $\longrightarrow$ Low Mass $\longleftarrow$ Medium Mass $\longleftarrow$ Heavy Mass

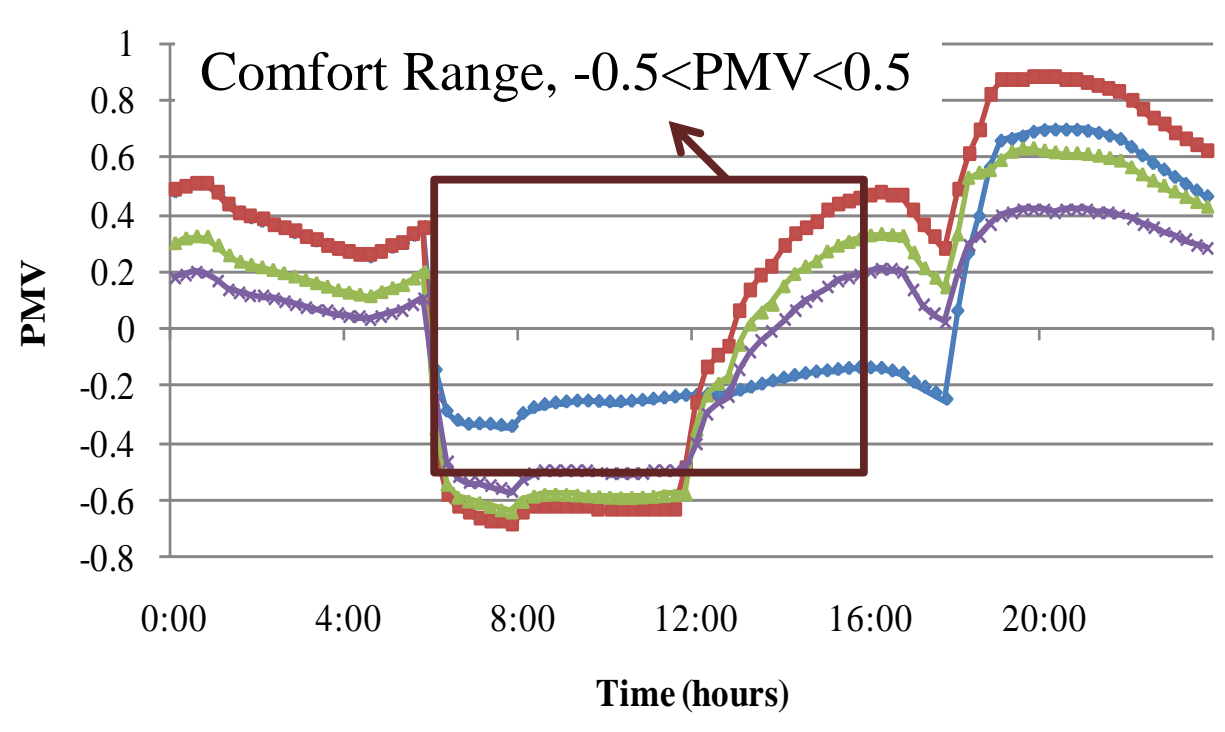

- PPD: Predicted Percentage of Dissatisfied

- PMV: Predicted Mean Vote 


\section{Scenario results with pre-cooling with exponential temperature reset}

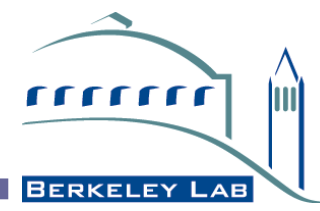

\begin{tabular}{|c|c|c|c|c|c|c|c|}
\hline \multirow{2}{*}{$\begin{array}{l}\text { Climate in } \\
\text { Summer }\end{array}$} & \multirow{2}{*}{$\begin{array}{c}\text { Thermal } \\
\text { Mass } \\
\text { Level }\end{array}$} & \multicolumn{2}{|c|}{$50,000 \mathrm{ft}^{2}$} & \multicolumn{2}{|c|}{$100,000 \mathrm{ft}^{2}$} & \multicolumn{2}{|c|}{$200,000 \mathrm{ft}^{2}$} \\
\hline & & $\begin{array}{c}12 \text { PM-3 } \\
\text { PM }\end{array}$ & $\begin{array}{c}3 \text { PM-6 } \\
\text { PM }\end{array}$ & $\begin{array}{c}12 \text { PM-3 } \\
\text { PM }\end{array}$ & $\begin{array}{c}3 \text { PM-6 } \\
\text { PM }\end{array}$ & $\begin{array}{c}12 \text { PM-3 } \\
\text { PM }\end{array}$ & $\begin{array}{c}3 \text { PM-6 } \\
\text { PM) }\end{array}$ \\
\hline \multirow{3}{*}{ Warm } & Low & $13.2 \%$ & $15.2 \%$ & $12.4 \%$ & $13.4 \%$ & $12.5 \%$ & $13.2 \%$ \\
\hline & Medium & $15.3 \%$ & $17.5 \%$ & $15.1 \%$ & $16.6 \%$ & $15.1 \%$ & $16.0 \%$ \\
\hline & Heavy & $16.2 \%$ & $19.2 \%$ & $17.1 \%$ & $18.4 \%$ & $17.2 \%$ & $18.6 \%$ \\
\hline \multirow{3}{*}{ Hot } & Low & $14.3 \%$ & $15.9 \%$ & $13.4 \%$ & $14.9 \%$ & $12.7 \%$ & $14.1 \%$ \\
\hline & Medium & $16.3 \%$ & $18.6 \%$ & $15.6 \%$ & $18.1 \%$ & $14.9 \%$ & $17.6 \%$ \\
\hline & Heavy & $18.0 \%$ & $21.2 \%$ & $17.2 \%$ & $20.9 \%$ & $19.2 \%$ & $20.5 \%$ \\
\hline \multirow{3}{*}{$\begin{array}{c}\text { Extremely } \\
\text { Hot }\end{array}$} & Low & $16.5 \%$ & $17.5 \%$ & $15.5 \%$ & $16.6 \%$ & $14.8 \%$ & $15.9 \%$ \\
\hline & Medium & $18.6 \%$ & $21.0 \%$ & $18.0 \%$ & $20.3 \%$ & $17.5 \%$ & $19.8 \%$ \\
\hline & Heavy & $21.0 \%$ & $24.0 \%$ & $20.3 \%$ & $23.4 \%$ & $20.0 \%$ & $22.9 \%$ \\
\hline
\end{tabular}




\section{Conclusions}

- Thermal mass levels: As expected, the peak demand savings rise with increased building thermal mass levels

- Climate Conditions: As outside temperature increases, magnitude of DR increases

- DR Control Strategies: The "pre-cooling with exponential temperature set up" control strategy achieved the greatest peak demand savings and the flattest afternoon electric load shape

- Zone Comfort: "Exponential temperature reset" yields the flattest demand profile in a typical commercial building 